\title{
BACKSCATTER OF ELECTROMAGNETIC WAVES \\ FROM A ROUGH LAYER
}

$$
\text { by }
$$

VIJAY RAJ KUMAR

B. S., Banaras Hindu University, 1961

\section{A MASTER'S THESIS}

submitted in partial fulfillment of the

$$
\text { requirements for the degree }
$$

$$
\text { MASTER OF SCIENCE }
$$

Department of Electrical Engineering

Kansas State University

Manhattan, Kansas

\section{6}

Approved by:

$$
\frac{\text { S. H. גman: }}{\text { Major Professor }}
$$


The author wishes to thank Dr. S. H. Durrani for his help and quidance during the preparation of this thesis. Thanks are also due to Dr. W. W. Koepsel and Mr. D. E. Kaufman for many useful suggestions. The assistance of Mr. Gary Alexander and Mr. John Cheng in the experimental work and of Mrs. Linda Toy and Mrs. Mary Lu Hill in typing the report is acknowledged. 
TABLE OF CONTENTS

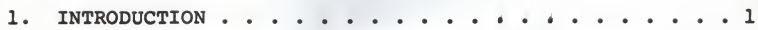

2. THE GENERAL KIRCHHOFF SOLUTION FOR SCATTERING

OF ELECTROMAGNETIC WAVES FROM ROUGH SURFACES . . . 7

2.0. General Formulation of the Problem. . . . . 7

2.1. Surface Rough in One Dimension. . . . . . 12

2.2. Surface Rough in Two Dimensions . . . . . . 18

3. BACKSCATTER OF ELECTROMAGNETIC WAVES FROM A ROUGH LAYER. . . . . . . . . . . . . . 23

3.0. E Fields in Various Regions ......... . 23

3.1. Radar Cross Section of Rough Layer. . . . . . 32

Case I: Short Pulse . . . . . . . . 34

Case II: Long Pulse . . . . . . . . . 39

3.2. Summary of Results. . . . . . . . . . 41

3.3. Discussion of Results . . . . . . . . 43

3.4. Notation. . . . . . . . . . 4 47

4. RADAR CROSS SECTION MEASUREMENTS FOR A ROUGH LAYER

BY ACOUSTIC SIMULATION TECHNIQUES. . . . . . . 50

4.0. Basic Concepts. . . . . . . . . . 50

4.1. Acoustic Simulator Components . . . . . . 52

4.2. Target Description. . . . . . . . . 55

4.3. Procedure for the Experiment. . . . . . . 58

4.4. Results . . . . . . . . . . . 60

4.5. Discussion of Results . . . . . . . . 66

5. Conclusions. . . . . . . . . . . 70

6. RECOMMENDATIONS FOR FUTURE STUDIES ......... 73

APPENDIX I - TARGET CONSTRUCTION, PROPERTIES \&

STATISTICS . . . . . . . . . . . . . 75

APPENDIX II - COMMENTS ON SIGNAL RECORDING; SAMPLE

Calculations . . . . . . . . . . . . 82 
Page

APPENDIX III - TABLES OF MEASURED DATA. . . . . . 86 APPENDIX IV - SCATTERING FROM PERIODICALLY ROUGH SURFACES. ................. 88 BIBLIOGRAPHY. . . . . . . . . . . . 90 


\section{LIST OF FIGURES}

Figure No.

Page

1. The scattering geometry. . . . . . . . . . . 8

2. Derivation of equation (7) . . . . . . . . . 8

3. The "local" scattering geometry. . . . . . . . . . . 11

4. The "scattering geometry" for rough layer. . . . . . . . 24

5. Further details of rough layer scatter . . . . . . . 28

6. The Acoustic Simulator. . . . . . . . . . . . 51

7. Plexiglas layer. . . . . . . . . . . . . 56

8. Normalized autocorrelation function for full length of rough surface of layer . . . . . . . . . . . 57

9. Normalized autocorrelation function for four-fifths length of rough surface of layer . . . . . . . . . 61

10. Radar cross section versus $\sigma_{z} / \lambda$ for rough back configuration of layer . . . . . . . . . . . . 62

11. Radar cross section versus angle of incidence for rough back configuration of layer. . . . . . . . . . . 63

12. Radar cross section versus $\sigma_{z} / \lambda$ for rough front configuration of layer . . . . . . . . . . . . 64

13. Radar cross section versus angle of incidence for rough front configuration of layer . . . . . . . . . 65

14. On backscatter calculations. . . . . . . . . . . 83 


\section{LIST OF TABLES}

Table No.

Page

I. Beamwidth of transducers. . . . . . . . . . . 53

II. Wavelength of sound in water. . . . . . . . . . . 77

III. Wavelength of sound in plexiglas. . . . . . . . . . 77

IV. Measured data for rough back configuration of layer . . 86

v. Measured data for rough front configuration of layer. . 87 


\section{INTRODUCTION}

The problem of reflection of electromagnetic waves from natural surfaces is of interest in a number of different fields. In particular, quite a few attempts have been made recently using the results of monostatic and bistatic radar reception of surfaces echoes to map the surface of terrestrial bodies $[28,29]$. In these experiments information about the surface to be mapped is obtained by comparing the target's echo signal with the hypothetical signal which would have been received from an ideal target. The data is then interpreted to determine the electrical and statistical properties of the target surface.

When an electromagnetic wave is incident on a plane interface (of sufficiently large dimensions) between two media, it is reflected specularly according to well known laws. The reflected field depends on the wavelength, the angle of incidence and the electromagnetic properties (permittivity, permeability and conductivity) of the two adjoining media. In fact, the laws of reflection by a plane boundary are so well understood that, conversely, the electrical properties of a material are often determined by measuring its reflection coefficient.

On the other hand, the incident field on a rough surface will be scattered into various directions, though certain privileged directions may receive more energy than others. Roughness itself is a relative term. The same surface may be rough for some wavelengths and smooth for others; or for the same wavelength it may be either rough or smooth for different angles of incidence. A smooth surface is thus the limiting case of a 
rough one. The limit depends on the wavelength and angle of incidence of the incident radiation.

The problem of scattering from a rough surface has become of special interest in recent years, particularly in connection with the propagation of radio waves at frequencies above $30 \mathrm{Mc} / \mathrm{s}$. A large number of papers $[1-2,4-6,14-19,40,41]$ have been published on the subject of scattering from rough surfaces, especially in the last 15 years. Extensive experimental data have been accumulated and many theories have been developed to explain and predict measured data. None of these theories are general and rigorous at the same time. In order to arrive at results that lend themselves to reasonably simple numerical calculations, or to arrive at any result at all, certain simplifying assumptions are introduced into these theories. By far, the largest number of rough surface scatter theories are based on the Kirchhoff approximation of the boundary conditions which are required to evaluate the Helmholtz integral. Apart from the original Kirchhoff postulation, other methods of approximating these boundary conditions have also been suggested, e.g. estimating the surface current distribution from the incident magnetic field, or expressing the total field by means of local reflection coefficients. In principle these approximations are but different versions of the same Kirchhoff method or the method of physical optics. Another approximation used in most theories is to assume the surface to be perfectly conducting. Within the errors caused by various approximations, the general features of the behaviour of electromagnetic radiation reflected by rough surfaces have thus become reasonably well known by now. 
Scope and Outline of Present Work.

The simplified approach for scatter from rough surfaces, described above, is discussed further in Chapter 2. However, this approach is inadequate for many cases of practical interest, because natural surfaces are neither perfectly conducting nor perfectly hard, so that the region beneath the surface inevitably contributes to the scattered signal. Therefore, a model consisting of a partially reflecting layer and taking into account the sub-surface region will predict the behaviour of a natural surface much better than the usual model consisting of a perfectly conducting rough surface. The problem thus requires the analysis of the backscatter of electromagnetic waves from a rough layer rather than a rough surface.

The most general case of layer scatter would have both the surfaces (boundaries) randomly rough, with no restriction on the mean depth. However, the solution of such a general case will be very complex. To avoid these complexities, certain simplifying assumptions are made. It is assumed that

(1) the mean depth of layer is much greater than the wavelength of the incident wave within the layer, and

(2) the front surface of layer is plane and the back surface randomly rough.

The restriction on the mean depth of layer is necessary to facilitate the calculation of the field produced at the front surface by the wave scattered by the back (rough) surface, by 
considering the field to be in the far region or Fraunhofer zone of diffraction. The second assumption simplifies the analysis of backscattered field. A layer having the front surface smooth and the back surface rough was chosen--rather than the opposite case of rough front and smooth back--because it is comparatively simpler to analyze the backscattered field in the former case.

A backscatter theory for the simplified rough layer is developed in Chapter 3. In spite of the simplifying assumptions, the considered rough layer does have a few applications, among others, in radar astronomy. In radar astronomy, the backscattered signal from the moon or other terrestrial bodies is analyzed in such a manner as to yield fundamental information regarding the nature of the target surface. Most of the visible side of the lunar surface consists of the so-called seas which have a relatively smooth, flat top surface. These seas are believed to be made up of some porous, sandy material which covers a rough rocklike inner crust. Therefore, the lunar surface may be taken to consist of a layer with smooth front and rough back, for which the theory developed in Chapter 3 is applicable.

\section{Assumptions and Restrictions.}

In developing the backscatter theory for the simplified rough layer, the Kirchhoff method is used with the following assumptions (see also Beckmann, Ref. 5, Ch. 1 \& 3):

(1) The dimensions of the scattering elements of the rough surface are taken as either much smaller or much greater than the wavelength of the incident radiation. 
(2) The radius of curvature of the scattering elements is taken to be much greater than the wavelength of the incident radiation.

(3) Shadowing effects are neglected.

(4) Multiple scattering is neglected.

(5) The incident wave is plane and linearly polarized with the $\vec{E}$ vector perpendicular to the plane of incidence.

(6) Only the far zone field is calculated, but no assumption is made as to its polarization. Any possible cross-polarized component has not been set to zero, but simply excluded from the results.

(7) The rough back of the layer is assumed to be in intimate contact with an infinitely thick slab of a perfectly conducting material. The roughness of the interface is described by a random variable corresponding to the interface-level fluctuations, and is assumed to have Gaussian statistics.

(8) The beamwidth of the incident wave is considered to be much smaller than the mean depth of the layer.

(9) The material of the layer is assumed to be lossless.

Experimental Work.

In order to obtain some direct backscatter measurements, an experimental study of the behaviour of a rough layer was undertaken. The acoustic simulation technique was used. The layer material chosen was plexiglas, since it. was one of the few materials available which had a comparatively low reflection coefficient (0.38) and was not affected by water. A high reflection coefficient 
would reduce the power transmitted into the layer, thus making the resultant scatter at the rough back too small to be easily detected. The experimental results are given in Chapter 4 . From the measured data, the following graphs were obtained:

(1) $\left\langle\sigma_{0}\right\rangle$ versus $\sigma_{z} / \lambda$, the back interface being rough.

(2) $\left\langle\sigma_{0}\right\rangle$ versus the angle of incidence, the back interface being rough.

(3) Same as (1), but with the front interface rough.

(4) Same as (2), but with the front interface rough. where

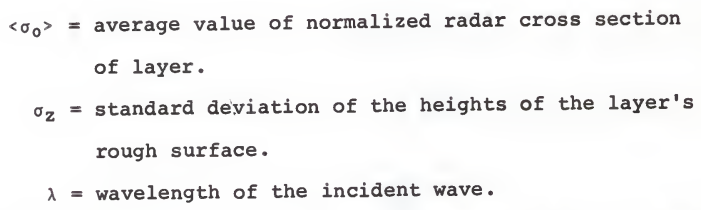

The frequencies used were: $0.72 \mathrm{Mc}, 1.00 \mathrm{Mc}, 1.28 \mathrm{Mc}, 1.60 \mathrm{Mc}$, $1.90 \mathrm{Mc}, 2.25 \mathrm{Mc}, 3.00 \mathrm{Mc}$, and $3.50 \mathrm{Mc}$. The angles of incidence were: $0^{\circ}, 5^{\circ}, 10^{\circ}$ and $20^{\circ}$.

The experimental results obtained are discussed in chapter 4. Only a partial attempt was made to check the experimental results of Chapter 4 with the theory developed in Chapter 3, but it is to be noted that the experiment violated assumptions (8) and (9) of the theory, which makes a direct comparison difficult. 
2. THE GENERAL KIRCHHOFF SOLUTION FOR SCATTERING OF ELECTROMAGNETIC WAVES FROM ROUGH SURFACES.

A large number of theories $[1-2,4-6,14-19,40-41]$ have been developed on the problem of scatter of electromagnetic waves from a rough surface. In this chapter a brief summary of the solution given by Beckmann [5] is presented, the same notation being used. In Chapter 3, this solution will be extended to cover backscatter from a rough layer. This chapter could not be made very short because the results derived here shall be used in Chapter 3 .

\subsection{General Formulation of the Problem.}

As shown in Fig. 1, the Cartesian coordinates $x, y, z$ with origin 0 and unit vectors $\vec{a}_{x^{\prime}} \vec{a}_{y^{\prime}} \vec{a}_{z}$ shall be used. Let the rough surface $s$ be given by the function

$$
\xi=\xi(x, y)
$$

where $\xi$ is a random variable.

The mean level of the surface is the plane

$$
z=0
$$

All quantities associated with the incident field will be denoted by the subscript 1 and those associated with the scattered field by the subscript 2. The medium in the space $z>\xi$ is assumed to be free space. The incident field $\vec{E}_{1}$ is assumed to be a harmonic plane wave of unit amplitude:

$$
E_{1}=e^{j\left(\vec{k}_{1} \cdot \vec{r}-\omega t\right)}
$$

where $\left|\vec{k}_{1}\right|=k=2 \pi / \lambda$, and $\vec{k}_{1}$ is the propagation vector, which will always lie in the $x z$ plane, and $r$ is the radius vector:

$$
\vec{r}=\vec{a}_{x}+y \vec{a}_{y}+z \vec{a}_{z} \text {. }
$$




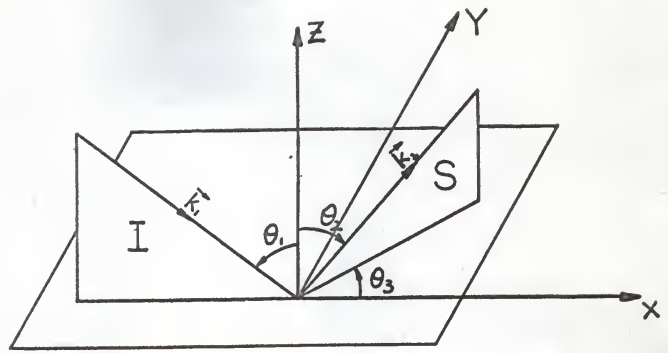

FIG.I. THE SCATTERING GEOMETRY.

$I=P L A N E$. OF INCIDENCE.

$S=$ THE SCATTERING PLANE.

$\theta_{1}=$ ANGLE OF INCIDENCE.

$\theta_{2}, \theta_{3}=$ SCATTERING ANGLE.

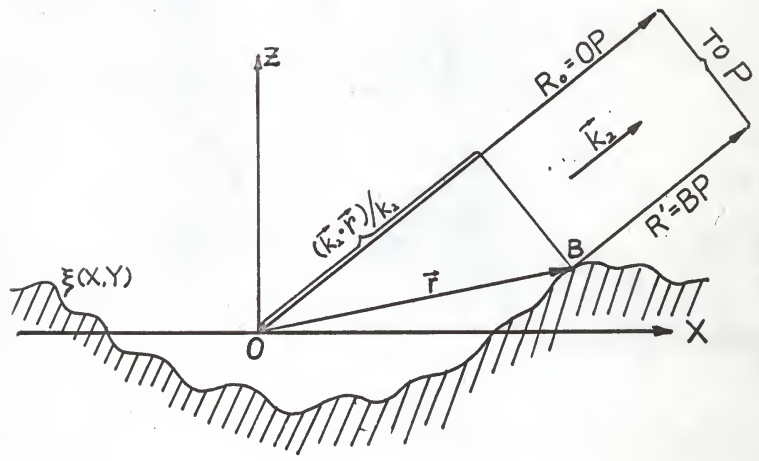

FIG.2. DERIVATION OF EQUATION (7). 
In particular, for points on the surface $S$, we have

$$
(\vec{r})_{s}=x \vec{a}_{x}+y \vec{a}_{y}+\xi(x, y) \vec{a}_{z}
$$

The angle of incidence, included between the direction of propagation of $\vec{E}_{1}$ and the $z$ axis, will be denoted by $\theta_{1}$. The scattering angle, included between $\vec{a}_{z}$ and $\vec{k}_{2}$, will be denoted by $\theta_{2}$, with $\theta_{1}$ and $\theta_{2}$ measured in opposite senses from the positive $z$ axis (Fig, 1). Here $\vec{k}_{2}$ is the reflected propagation vector, lying in the $x z$ plane with

$$
\left|\vec{k}_{2}\right|=\left|\vec{k}_{1}\right|=k=2 \pi / \lambda \text {. }
$$

For lateral scattering out of the plane of incidence $\left(\vec{k}_{1}, \vec{a}_{z}\right)$, a further angle $\theta_{3}$ is introduced (Fig. 1).

The time factor $\exp (-j \omega t)$ will hence forward be suppressed. The polarization of $\vec{E}_{1}$ shall be termed vertical if $\vec{E}_{1}$ lies in the plane of incidence $\left(\vec{k}_{1}, \vec{a}_{z}\right)$, and again the scattered field $\vec{E}_{2}$ will be called vertically polarized if it lies in the scattering plane $\left(\vec{k}_{2}, \vec{a}_{z}\right)$. Similarly, in horizontal polarization, $\overrightarrow{\mathrm{E}}_{1}$ and $\vec{E}_{2}$ are normal to the incidence plane and the scattering plane respectively. The quantities associated with vertical polarization will be denoted by the superscript "+" and those associated with horizontal polarization by the superscript "-"

The scalar value of the scattered field $\mathrm{E}_{2}$ at the point of observation $\mathrm{P}$ is given by the Helmholtz integral

$$
E_{2}(P)=\frac{1}{4} \iiint_{S}\left(E \frac{\partial \psi}{\partial n}-\psi \frac{\partial E}{\partial n}\right)_{S} d s
$$


where

$$
\Psi=\frac{e^{j k 2 R^{\prime}}}{R^{\prime}}
$$

and $R^{-}$is the distance from $P$ to a point $\vec{I}$ on the surface $S$.

No assumption is made as to the polarization of $\vec{E}_{2}(P)$; any possible cross-polarized component has not been set equal to zero, but simply excluded from the present range of interest.

In order to deal with plane scattered waves rather than spherical ones, we let $R^{\prime} \rightarrow \infty$, i.e., we remove $P$ to the Fraunhofer zone of diffraction; then as will be seen from Fig. 2 ,

$$
\mathrm{k}_{2} \mathrm{R}^{a}=\mathrm{k}_{2} \mathrm{R}_{0}-\overrightarrow{\mathrm{k}}_{2} \cdot \overrightarrow{\mathrm{r}}
$$

where $R_{0}$ is the distance of $P$ from the origin, so that

$$
\Psi=\frac{e^{j k_{2} R_{0}-j \vec{k}_{2} \cdot \vec{r}}}{R_{0}} .
$$

In Eq. (5), E and $\frac{\partial E}{\partial n}$ are the field and its normal derivative on $\mathrm{S}$. The exact value of these two quantities is in general unknown and the Kirchhoff or physical optics method consists essentially in approximating the values of $E$ and $\frac{\partial E}{\partial n}$ on $S$ and then evaluating the integral (5).

In the present case the field at any point of the surface shall be approximated by the field that would be present on the tangent plane at that point. The radius of curvature of the irregularities on the surface is assumed large compared with the wavelength of the incident field. Within this approximation the field and its normal derivative on $\mathrm{S}$ will be:

$$
\begin{aligned}
(E)_{S} & =(1+R) E_{1} \\
\left(\frac{\partial E}{\partial n}\right)_{S} & =(\nabla E \cdot \vec{n})_{S}=j(l-R) E_{1} \vec{k}_{1} \cdot \vec{n}
\end{aligned}
$$




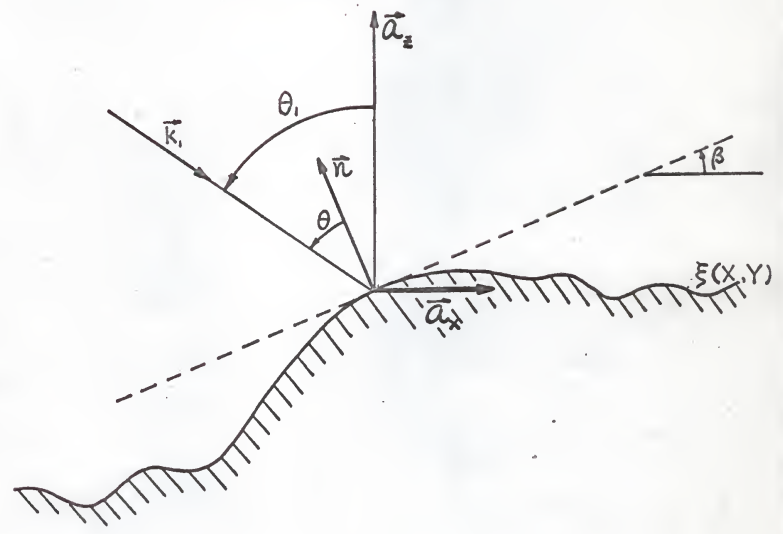

$\theta$ IS THE "LOCAL" ANGLE OF INCIDENCE WITH RESPECT TO THE NORMAL $\vec{n}$ AT THE CONSIDERED POINT.

$\theta_{1}$ IS THE "OVERALL" aNGLE OF INCIDENCE DEFINED WITH RESPECT TO $\vec{a}_{z} ; I^{\circ}$ IS CONSTANT FOR THE WHOLE SURFACE

fig. 3. THE "LOCAL" SCATTERING geOMETRY. 
where $\vec{n}$ is the normal to the surface at the considered point and $R$ is the reflection coefficient of a smooth plane. The reflection coefficient $R$ depends not only on the angle of incidence and the electrical properties of the reflecting material, but also on the polarization of the incident wave. The Fresnel reflection coefficient for a smooth plane are

$$
\begin{aligned}
& \mathbf{R}^{+}=\frac{Y^{2} \cos \theta-\sqrt{ }\left(Y^{2}-a \sin ^{2} \theta\right)}{Y^{2} \cos \theta+\sqrt{ }\left(Y^{2}-a \sin ^{2} \theta\right)} \\
& R^{-}=\frac{\cos \theta-\sqrt{ }\left(Y^{2}-a \sin ^{2} \theta\right)}{\left.\cos \theta+\sqrt{ } Y^{2}-a \sin ^{2} \theta\right)}
\end{aligned}
$$

where $a=1 / \mu_{r}^{2}$ and $\theta$ is the local angle of incidence (Fig. 3 ). The quantity $Y$ is the normalized admittance of the medium in the space $z<\xi$ and is given by

$$
Y=\varepsilon_{r} / \mu_{r}
$$

where $\varepsilon_{r}$ is the relative complex permittivity:

$$
\varepsilon_{r}=\varepsilon / \varepsilon_{0}+j 60 \lambda \sigma
$$

with $\varepsilon$ the dielectric constant, $\sigma$ the conductivity; and $\mu_{r}=\mu_{\mu_{0}}$ is the relative permeability which may have an imaginary part if the medium has magnetic losses. The free space electromagnetic constants are denoted by $\varepsilon_{0}$ and $\mu_{0}$.

\subsection{Surface Rough in One Dimension.}

Consider a surface rough in one dimension only. This surface will be constant along the $y$ coordinate, so that $\vec{n}$ will always be in the plane of incidence $(x, z)$. Then 


$$
\xi(x, y)=\xi(x)
$$

and $\theta=\theta_{1}-\beta=\theta_{1}-\arctan \xi^{\circ}(x)$.

Substituting (8), (9) and (10) in (5) we get

$$
E_{2}=\frac{j e^{j k R}}{4 \pi R_{0}} \int_{S}(\overrightarrow{R v}-\vec{p}) \cdot \vec{n} e^{j \vec{v} \cdot \vec{r}} d s
$$

where

$$
\begin{aligned}
\vec{v} & =\vec{k}_{1}-\vec{k}_{2}=k\left[\left(\sin \theta_{1}-\sin \theta_{2}\right) \vec{a}_{x}-\left(\cos \theta_{1}+\cos \theta_{2}\right) \vec{a}_{z}\right] \\
& =v_{x} \vec{a}_{x}+v_{z} \vec{a}_{z} \\
\vec{p} & =\vec{k}_{1}+\vec{k}_{2}=k\left[\left(\sin \theta_{1}+\sin \theta_{2}\right) \vec{a}_{x}+\left(\cos \theta_{2}-\cos \theta_{1}\right) \vec{a}_{z}\right] \\
\vec{n} & =-\sin \beta \vec{a}_{x}+\cos \beta \vec{a}_{z} \\
\vec{r} & =x \vec{a}_{x}+\xi(x) \vec{a}_{z} \\
d s & =\sec \beta d_{x} \\
\tan \beta & =\xi^{\prime}(x) .
\end{aligned}
$$

For a surface extending from $x=-I$ to $x=+L$, (17) reduces to

$$
E_{2}=\frac{j k e^{j k R_{0}}}{4 \pi R_{0}} \int_{-L}^{+L}\left(a \xi^{-}-b\right) e^{j\left(v_{x} x+v_{z} \xi\right)} d x
$$

where

$$
\begin{aligned}
& a=(1-R) \sin \theta_{1}+(1+R) \sin \theta_{2} \\
& b=(1+R) \cos \theta_{2}-(1-R) \cos \theta_{1} .
\end{aligned}
$$

Define a scattering coefficient:

$$
\rho=\frac{E_{2}}{E_{20}}
$$


where $E_{20}$ is the field reflected in the direction of specular reflection $\left(\theta_{2}=\theta_{1}\right)$ by a smooth, perfectly conducting plane of the same dimensions under the same angle of incidence at the same distance, when the incident wave is horizontally polarized.

For a smooth perfectly conducting plane (18) reduces to

$$
E_{20}=\frac{j k e^{j k R_{0}} L \cos \theta 1}{\pi R_{0}} \text {. }
$$

From (18), (21) and (22)

$$
\rho=\frac{1}{4 L \cos \theta} \int_{-L}^{+L}\left(a \xi^{-}-b\right) e^{j\left(v_{x} x+v_{z} \xi\right)} d x .
$$

The integral (23) is easily evaluated when a and b are constants, but not in other cases. One way of solving the general case is to average $R$ over the surface, making $a$ and $b$ independent of $x$. The other, more important case is $\mathrm{Y} \rightarrow \infty$ or a perfectly conducting surface.

Equations (11) and (12) then give

$$
\mathbf{R}^{+}=1, \mathbf{R}^{-}=-1
$$

so that $\mathrm{a}$ and $\mathrm{b}$ in (19) and (20) are independent of $\mathrm{x}$. Integrating by parts, (23) yields

$$
\rho \pm\left(\theta_{1} ; \theta_{2}\right)= \pm \sec \theta_{1} \frac{1+\cos \left(\theta_{1}+\theta_{2}\right)}{\cos \theta_{1}+\cos \theta_{2}} \frac{1}{2 L} \int_{-L}^{+L} e^{j \vec{v} \cdot \vec{r}} d x+\frac{e^{ \pm}(L)}{2 L}
$$

where

with

$$
e^{ \pm}(L)=\left.\frac{j \sec \theta_{1} \sin \theta \pm}{k\left(\cos \theta_{1}+\cos \theta_{2}\right)} e^{j \vec{v} \cdot \vec{r}(x)}\right|_{-L} ^{+L}
$$

$$
\theta^{+}=\theta_{2}, \theta^{-}=\theta_{1} .
$$


Formula (24) is the general solution for a perfectly conducting, one dimensionally, rough surface.

For $L>>\lambda$, the second term of (24) is negligible compared to the first, so (24) reduces to:

$$
\rho \pm= \pm \frac{F_{2}}{2 L} \int_{-L}^{+L} e^{j \vec{v} \cdot \vec{r}} d x
$$

with

$$
F_{2}\left(\theta_{1}, \theta_{2}\right)=\sec \theta_{1} \frac{1+\cos \left(\theta_{2}+\theta_{2}\right)}{\cos \theta_{1}+\cos \theta_{2}} \text {. }
$$

Assume $\xi(x)$ to be a random stationary process with a mean value $\langle\xi(x)\rangle=0$, where the angular brackets $<$ denote the mean value.

Representing the complex conjugate quantity by an asterisk, and using $(26)$, the mean square scatter coefficient $\langle\rho \rho *\rangle$ is given by :

$$
\langle\rho \rho *\rangle=\frac{F_{2}}{4 I^{2}} \int_{-I}^{+L} \int_{-L}^{+L} e^{j v_{x}\left(x_{1}-x_{2}\right)}<e^{j v_{2}\left(\xi_{1}-\xi_{2}\right)}>d x_{1} d x_{2}
$$

where

$$
\xi_{1}=\xi\left(x_{1}\right) \text { and } \xi_{2}=\xi\left(x_{2}\right)
$$

and

$$
\begin{gathered}
e^{j v_{z}\left(\xi_{1}-\xi_{2}\right)}=\int_{-\infty}^{+\infty} \int_{-\infty}^{+\infty} w\left(z_{1}, z_{2}\right) e^{j v_{z}\left(\xi_{1}-\xi_{2}\right)} d z_{1} d z_{2} \\
=x_{2}\left(v_{z},-v_{z}\right)
\end{gathered}
$$

is the two dimensional characteristic function of the distribution $\mathrm{W}\left(\mathrm{z}_{1}, \mathrm{z}_{2}\right)$. 
Introduce a separation parameter, defined by

$$
\tau=x_{1}-x_{2} \text { 。 }
$$

Substituting equations (29) and (30) in (28), and after elementary manipulations, we get

$$
\left\langle\rho \rho *=\frac{F_{2}{ }^{2}}{2 I} \int_{-L}^{+L} e^{j v_{x} \tau}\left[x_{2}\left(v_{z},-v_{z}\right)\right] d \tau .\right.
$$

Assume $z_{1}$ and $z_{2}$ are normally distributed with mean values zero, variances $\sigma_{z}{ }^{2}$ and correlated by a correlation coefficient $C(\tau)$; then

$$
\mathrm{W}\left(\mathrm{z}_{1}, \mathrm{z}_{2}\right)=\frac{1}{2 \pi \sigma_{z}{ }^{2} \sqrt{ }\left(1-\mathrm{C}^{2}\right)} \exp \left[-\frac{\mathrm{z}_{1}{ }^{2}-2 C z_{1} z_{2+z_{2}}{ }^{2}}{2 \sigma \mathrm{z}^{2}\left(1-\mathrm{C}^{2}\right)}\right]
$$

The characteristic function for the above distribution is given by

$$
x_{2}\left(v_{z},-v_{z}\right)=\exp \left[-v_{z}^{2} \sigma_{z}^{2}(1-C)\right]
$$

Choose a Gaussian correlation coefficient

$$
C(\tau)=e^{-\tau^{2} / T^{2}}
$$

where $T$ is the correlation distance for which $C(\tau)$ will drop to the value $e^{-1}$. It is to be noted that a surface with a given correlation distance $T$, appears to be randomly rough to an incident beam only if the beamwidth is large compared to $T$ (i.e., the area illuminated is large compared to $\mathrm{T}^{2}$ ); for in the contrary case the beam will not cover a randomly rough surface with enough variations to justify a statistical description, but will cover just one or two irregularities. 
Substituting (34) in (33) and expanding it in an exponential series, we get

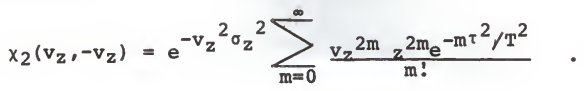

For briefness let

$$
v_{g}=v_{z} \sigma_{z}=\frac{2 \pi \sigma_{z}}{\lambda}\left(\cos \theta_{1}+\cos \theta_{2}\right) .
$$

Substituting (35) and (36) in (31), gives

$$
\left\langle\rho \rho^{*}\right\rangle=\frac{F_{2}}{2 L} e^{-g} \sum_{m=0}^{\infty} \frac{q^{m}}{m} \int_{-L}^{+L} e^{j v_{X} \tau-m \tau^{2} / T^{2}} d \tau .
$$

In order to avoid a divergent integral it is necessary to rearrange (37) by noting that

$$
\langle\rho \rho *\rangle=\langle\rho\rangle\langle\rho *\rangle+D\{\rho\}
$$

where

$$
D\{\rho\}=|\rho-\langle\rho\rangle|^{2}
$$

is the variance of the complex variable $\rho$.

This variance $D\{\rho\}$ may be written in terms of an integral over the scattering surface as

$$
D(\rho)=\frac{F_{2}}{2 L} \int_{-L}^{+L} e^{j v_{x} \tau}\left[x_{2}\left(v_{z},-v_{z}\right)-x\left(v_{z}\right) x^{*}\left(v_{z}\right)\right] d \tau
$$

where

$$
\begin{gathered}
\left\langle e^{j v_{z} \xi_{1}}\right\rangle=\left\langle e^{\left.j v_{z} \xi_{2}\right\rangle}=\left\langle e^{j v \xi}\right\rangle=\left\langle e^{j v_{z} z}\right\rangle\right. \\
=\int_{-\infty}^{+\infty} w(z) e^{j v_{z} z} d z=x\left(v_{z}\right)
\end{gathered}
$$


is the characteristic function associated with the one dimensional probability function $W(z)$ for the variable $z$.

Since $z_{1}$ and $z_{2}$ are normally distributed, therefore $z$ is also normally distributed with the same variance $\sigma_{z}{ }^{2}$ and its distribution function is given by

$$
W(z)=\frac{1}{\sigma_{z} \frac{1}{(2 \pi)}} e^{-z^{2} / 2 \sigma_{z}^{2}} .
$$

The characteristic function associated with the above distribution is

$$
x\left(v_{z}\right)=\exp \left(-\frac{1}{2} \sigma_{z}{ }^{2} v_{z}{ }^{2}\right)=\exp \left(-\frac{1}{2} g\right) .
$$

Substituting (41) in (26), we get

$$
\langle\rho\rangle=x\left(v_{z}\right) \rho_{0}
$$

where

$$
\rho_{0}=\frac{\operatorname{sinv} v_{x} L}{v_{x}^{L}}
$$

Replace the limits of integration in (37) and (40) from $\pm \mathrm{L}$ to $\pm^{\infty} ;$ this is permissible since the integral receives significant contributions only from the region near $\tau=0$. Substituting (35), (36), (43), (44) in (38) and evaluating we get

$$
\left\langle\rho \rho^{*}\right\rangle=e^{-g}\left[\rho_{0}{ }^{2}+\frac{\sqrt{ } \pi T F_{2}{ }^{2}}{2 L} \sum_{m=1}^{\infty} \frac{q^{m}}{m ! J_{m}} e^{-v x^{2} T^{2} / 4 m}\right] .
$$

Note that

$$
\langle\rho \rho *\rangle=\rho^{2}{ }_{\mathrm{RMS}}=\frac{\left\langle\mathrm{E}_{2} \mathrm{E}_{2}^{*}\right\rangle}{\left|\mathrm{E}_{20}\right|^{2}} .
$$

\subsection{Surface Rough in Two Dimensions.}

Repeating the procedure of one dimensional rough surface, the scatter coefficient $\rho$ for a surface $\xi(x, y)$ rough in two di- 
mensions is given by

$$
\rho=\frac{E_{2}}{E_{20}}=\frac{1}{4 X Y \cos \theta_{1}} \int_{-X}^{+X} \int_{-Y}^{+Y}\left(a \xi_{x}+c \xi_{y}^{-}-b\right) e^{j \vec{v} \cdot \vec{r}} d x d y
$$

where

$$
E_{20}=\frac{j k e^{j k R_{0}} \cos \theta_{1}}{2 \pi R_{0}}
$$

$A=4 X Y$ is the area of $S$ projected into the $x y$ plane.

$\vec{v}=k\left[\left(\sin \theta_{1}-\sin \theta_{2} \cos \theta_{3}\right) \vec{a}_{x}-\sin \theta_{2} \sin \theta_{3} \vec{a}_{y}-\left(\cos \theta_{1}+\cos \theta_{2}\right) \vec{a}_{z}\right]$

$$
=v_{x} \vec{a}_{x}+v_{y} \vec{a}_{y}+v_{z} \vec{a}_{z}
$$

$a=(1-R) \sin \theta_{1}+(1+R) \sin \theta_{2} \cos \theta_{3}$

$b=(1+R) \cos \theta_{2}-(1-R) \cos \theta_{1}$

$c=(1+R) \sin \theta_{2} \sin \theta_{3}$

For a perfectly conducting surface

$$
\mathbf{R}^{+}=1, \mathbf{R}^{-}=-1
$$

and $a, b, c$ are constants.

Integrating by parts $(46)$ reduces to

$$
\rho{ }^{ \pm}\left(\theta_{1} ; \theta_{2}, \theta_{3}\right)= \pm \frac{F_{3}}{A} \int_{-X}^{+X} \int_{+Y}^{+Y} e^{j \vec{v} \cdot \vec{r}} d x d y+e \frac{(X, Y)}{A}
$$

where

$$
\begin{aligned}
F_{3} & =\frac{1+\cos \theta_{1} \cos \theta_{2}-\sin \theta_{1} \sin \theta_{2} \cos \theta_{3}}{\cos \theta_{1}\left(\cos \theta_{1}+\cos \theta_{2}\right)} \\
e(X, Y) & =-\left.\frac{j c}{V_{Z}} \int_{-X}^{+X} e^{j \vec{v} \cdot \vec{r}}\right|_{-Y} ^{+Y} d x-\left.\frac{j a}{v_{Z}} \int_{-Y}^{+Y} e^{j \vec{v} \cdot \vec{r}}\right|_{-X} ^{+X} d Y .
\end{aligned}
$$

Equation (53) represents the edge effect term and is negligible compared to the first term of (52) when $A>>\lambda^{2}$; then (52) 
reduces to

$$
p^{ \pm}= \pm \frac{F_{3}}{A} \iint_{A}^{j \vec{v} \cdot \vec{r}} d x d y
$$

Assuming $\xi(x, y)$ to be a random stationary process with mean value $\langle\xi(x, y)\rangle=0$, the mean square scatter coefficient for a surface rough in two dimensions is given by $\left\langle\rho p^{*}\right\rangle$ and is found by using (54):

$$
\left\langle\rho \rho^{*}\right\rangle=\frac{F_{3}{ }^{2}}{A^{2}} \int_{-X}^{+X} \int_{-X}^{+X} \int_{-Y}^{+Y} \int_{-Y}^{+Y} \exp \left[j v_{X}\left(x_{1}-x_{2}\right)+j v_{Y}\left(y_{1}-y_{2}\right)\right]<e^{j v}\left(\xi 1-\xi_{2}\right)>x
$$

where $\xi_{1}=\xi\left(x_{1}, Y_{1}\right), \xi_{2}=\xi\left(x_{2}, Y_{2}\right)$.

Introduce a separation parameter $\tau$ defined as

$$
\tau=\left[\left(x_{2}-x_{1}\right)^{2}+\left(y_{2}-y_{1}\right)^{2}\right]^{\frac{1}{2}} .
$$

Substituting (56) in (55) and carrying out elementary manipulations, (55) reduces to

$$
\left\langle\rho \rho^{*}\right\rangle=\frac{2 \pi F_{3}{ }^{2}}{A} \int_{0}^{\infty} J_{0}\left(v_{x y} \tau\right)<e^{j v_{z}\left(\xi_{1}-\xi_{2}\right)}>\tau d \tau
$$

where $v_{x y}=\left(v_{x}{ }^{2}+v_{y}{ }^{2}\right)^{\frac{1}{2}}=k\left[\left(\sin \theta_{1}-\sin \theta_{2} \cos \theta_{3}\right)^{2}+\left(\sin \theta_{2} \sin \theta_{3}\right)^{2}\right]^{\frac{1}{2}}$

and $J_{0}\left(v_{x y} \tau\right)$ is a Bessel function of the first kind and order zero with argument $v_{x y}{ }^{\tau}$. Since only the regions near $\tau=0$ contribute to the integral and the contribution from $(X, Y)$ to $(\infty, \infty)$ is negligible, the range of integration in (57) has, for convenience, been made infinite. 
To avoid a divergent integral (57) is substituted in (38) to give the variance

$$
D\{p\}=\frac{2 \pi F_{3}{ }^{2}}{A} \int_{0}^{\infty} J_{0}\left(v_{x y} \tau\right)\left[x_{2}\left(v_{z},-v_{z}\right)-x\left(v_{z}\right) x^{*}\left(v_{z}\right)\right] \tau d \tau .
$$

Substituting (35), (36), (41), (43) in (59), we get

$$
\begin{aligned}
& D\{\rho\}=\frac{2 \pi F_{3}{ }^{2}}{A} \int_{0}^{\infty} J_{0}\left(v_{x y} \tau\right) \\
&\left.e^{-g} \sum_{m=0}^{\infty} \frac{g^{m}}{m !} e^{-m \tau^{2} / T^{2}}-e^{-g}\right] \tau d \tau \\
&= \frac{2 \pi F_{3}^{2}}{A} e^{-g} \sum_{m=1}^{\infty} \frac{g^{m}}{m !} \int_{0}^{\infty} J_{0}\left(v_{x y} \tau\right) e^{-m \tau^{2} / T^{2}} \tau d \tau
\end{aligned}
$$

The last expression was obtained by the interchange of integration and summation.

Since $\mathrm{m}>0$, we have

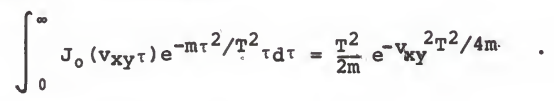

Substituting (61) in (60) gives

$$
D\{\rho\}=\frac{\pi F_{3}{ }^{2} T^{2}}{A} e^{-g} \sum_{m=1}^{\infty} \frac{q^{m}}{m ! m} e^{-v_{x y}{ }^{2} T^{2} / 4 m} .
$$

Substitution (62) in (38) gives

$$
\left\langle\rho \rho^{*}\right\rangle=e^{-g}\left[\rho_{0}^{2}+\frac{\pi T^{2} F_{3}^{2}}{A} \sum_{m=1}^{\infty} \frac{g^{m}}{m ! m} e^{-v Y_{x y}^{2} T^{2} / 4 m}\right] \text {. }
$$

Note that

$$
\left\langle\rho \rho^{*}\right\rangle=\rho_{\mathrm{RMS}}^{2}=\frac{\left\langle\mathrm{E}_{2} \mathrm{E}_{2}{ }^{*}\right\rangle}{\left|\mathrm{E}_{20}\right|^{2}}
$$

and in (63a),

$$
\rho_{0}=\frac{\sin v_{x} X \sin v_{y} Y}{v_{x} V_{y} X Y}
$$


Expressions have been derived for the mean scattered power $\left\langle p p^{\star}\right\rangle$ for a statistically rough surface in Eqs. (45a) and (63a), for roughness in one and two dimensions, respectively. 


\section{BACKSCATTER OF ELECTROMAGNETIC WAVES}

FROM A ROUGH LAYER

The case of backscatter of electromagnetic waves from a rough layer, with a plane upper boundary and a statistically rough lower boundary, is considered in this chapter. The geometry is shown in Fig. 4. The numbers 1,2 and 3 are used to denote, respectively, the initial medium through which the incident wave travels, the layer, and the medium which lies on the other side of the lower boundary (Fig. 4). The terms "front surface" and "back surface" shall be used interchangeably with "upper boundary and "lower boundary," respectively.

\subsection{E Fields in Various Regions}

It is assumed that mediums 1 and 2 consist of some lossless dielectric materials having the electromagnetic constants $\varepsilon_{1}$ ' $\mu_{1}$ and $\varepsilon_{2}, \mu_{2}$, respectively. Medium 3 is assumed to consist of a perfectly conducting material. The wavelength in medium 1 is given by $\lambda_{1}$ and in medium 2 by $\lambda_{2}$.

The results of this chapter have been derived under the restrictions of chapter 2, as given by Beckmann [5]. A list of the notation used is given at the end of the chapter.

The Cartesian coordinates $x, y, z$ with origin 0 at middle point of illuminated area on lower boundary (rough) of layer shall be used (Fig. 4). The rough boundary will be given by either of the two functions

$$
\begin{aligned}
& \xi=\xi(x) \\
& \xi=\xi(x, y)
\end{aligned}
$$




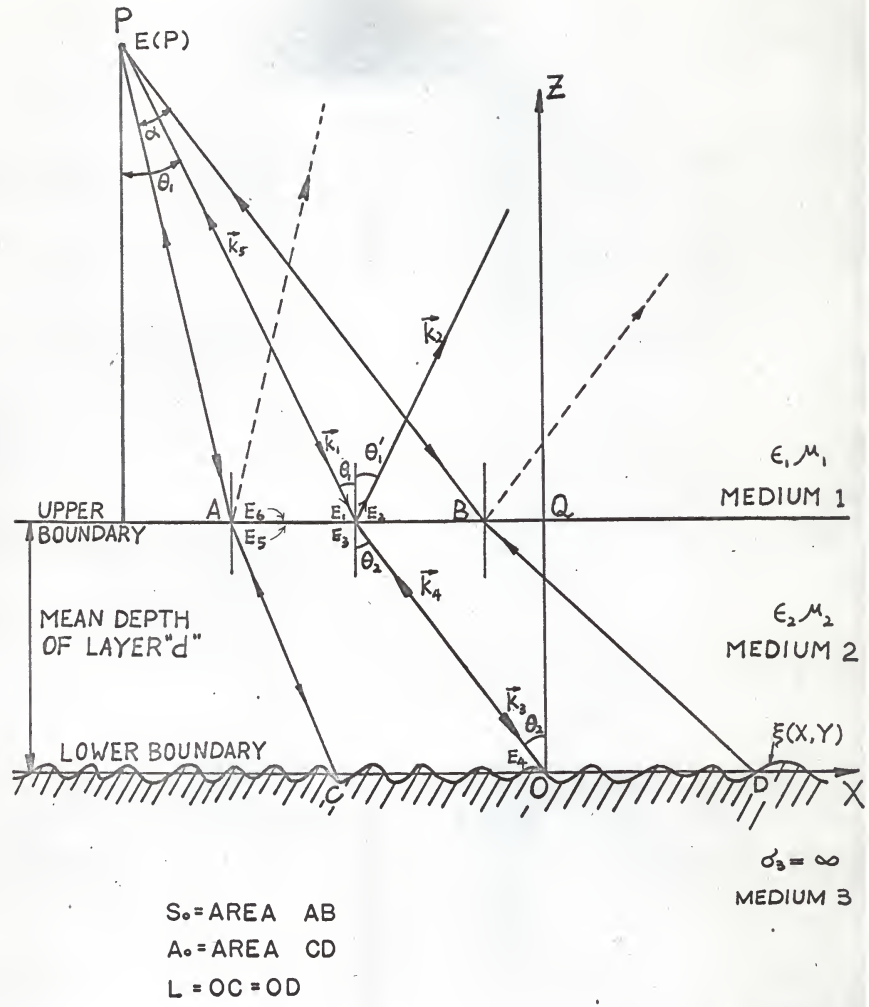

fIG.4. THE "SCATTERING GeOMETRY" FOR ROUGH LaYer. 
depending on whether the roughness is in one or two dimensions. The mean level of this boundary is the plane $z=0$ and the mean depth of the layer will be denoted by $d$.

It is assumed that the source of the incident wave is a radar situated at a point $P$ in medium 1 , such that the layer is at a large distance from it, in the Fraunhofer zone of diffraction (Fig. 4). If the beamwidth of the radar is small enough to make $\mathrm{s}_{1} \ll \mathrm{R}_{1}{ }^{2}$ (Fig. 5), then the electromagnetic wave incident on the upper boundary of the layer (the plane boundary) may be considered to consist of a plane harmonic wave.

The incident wave on the upper boundary of the layer is plane and linearly polarized with the $\vec{E}$ vector either in the plane of incidence $(x z)$ or perpendicular to it, and is given by

$$
E_{1}= \begin{cases}E_{0} e^{j\left(\vec{k}_{1} \cdot \vec{r}-\omega t\right)} & \text { over the beamwidth } \\ 0 & \text { elsewhere }\end{cases}
$$

where $\vec{k}_{1}$ is the propogation vector of magnitude $k_{1}=2 \pi / \lambda_{1}$ which will always $l i e$ on the $x z$ plane, and $\vec{r}$ is the radius vector

$$
\vec{r}=x \vec{a}_{x}+y \vec{a}_{y}+z \vec{a}_{z}
$$

In particular, for points on the upper boundary of the layer, we have

$$
\left.\vec{r}\right|_{S_{0}}=x \vec{a}_{x}+y \vec{a}_{y}+d \vec{a}_{z}
$$


A part of the energy within the incident beam will be reflected by the upper boundary of the layer and a part will be refracted into the layer (Fig. 4).

The angle of incidence included between the direction of propogation of $\mathrm{E}_{1}$ and the direction of the $\mathrm{z}$ axis will be denoted by $\theta_{1}$, the angle of reflection by $\theta^{\prime}{ }_{1}$, and the angle of refrattion, included between the $\mathrm{z}$ axis and $\vec{k}_{3}$, will be denoted by ${ }^{\prime}{ }^{\prime}$ where $\vec{k}_{3}$, with a magnitude

$$
\mathrm{k}_{3}=2 \pi / \lambda_{2}
$$

is the propogation vector of the transmitted wave within the layer.

Angles $\theta_{1}$ and $\theta_{2}$ are related as follows:

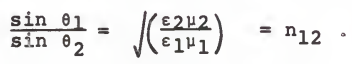

The beamwidth of the radar is given by the angle $\alpha$. Then the edges of the beam (half power points) make angles $\theta \pm \alpha / 2$ with the direction of the $\mathbf{z}$ axis.

The time factor $\exp (-j \omega t)$ will henceforward be suppressed. The reflection of the incident wave from the upper boundary will be in the specular direction, since the boundary is assumed to be plane. Therefore, this reflected energy will not be received at the radar, except in the case of normal incidence. On the upper boundary, the reflected electric field $E_{2}$ will be given by

$$
E_{2}=v_{12} E_{o} e^{j \vec{k}_{2} \cdot \vec{r}}
$$

where $v_{12}$ is the reflection coefficient at the upper boundary for 
a wave travelling from medium 1 into medium 2, and is given by or (12), chapter 2 .

$\vec{r}$ is given by $(5)$, and $\vec{k}_{2}$, with a magnitude

$$
k_{2}=2 \pi / \lambda_{1} \quad,
$$

is the propogation vector of the field reflected at the upper boundary.

The electric field $E_{3}$ of the refracted wave, just below the upper boundary is given by

$$
E_{3}=\frac{\cos \phi_{1}}{\cos \phi_{2} D_{12} E_{o} e^{j \vec{k}_{3} \cdot \vec{r}}}
$$

where

$$
\mathrm{D}_{12}=1+\mathrm{v}_{12}
$$

is the transmission coefficient at the upper boundary between media 1 and 2 for a wave travelling from medium 1 into medium 2 , $k_{3}$ is defined by (7), and the term $\frac{\cos \phi_{1}}{\cos \phi_{2}}=\frac{A B^{\prime}}{A^{\prime} B}$ (See Fig. 5) takes into account the decrease in intensity of $E_{3}$ due to broadening of the incident beam upon refraction. Fig. 5 defines $\phi_{1}$ and $\phi_{2}$. The cross sectional area of the beam $\mathrm{CD}^{\prime}$ at the lower boundary of the layer will be greater than the cross sectional area $A^{\prime} B$ at the upper boundary, since it is a divergent beam. Therefore, the intensity of the electric field $E_{4}$, incident on the lower boundary of layer will be reduced by the factor $\frac{A^{\prime} B}{C^{\prime}}=\frac{A B \cos \phi_{2}}{C D \cos \phi_{3}}$, as compared to the intensity of $E_{3}$. Then, $E_{4}$ is given by 


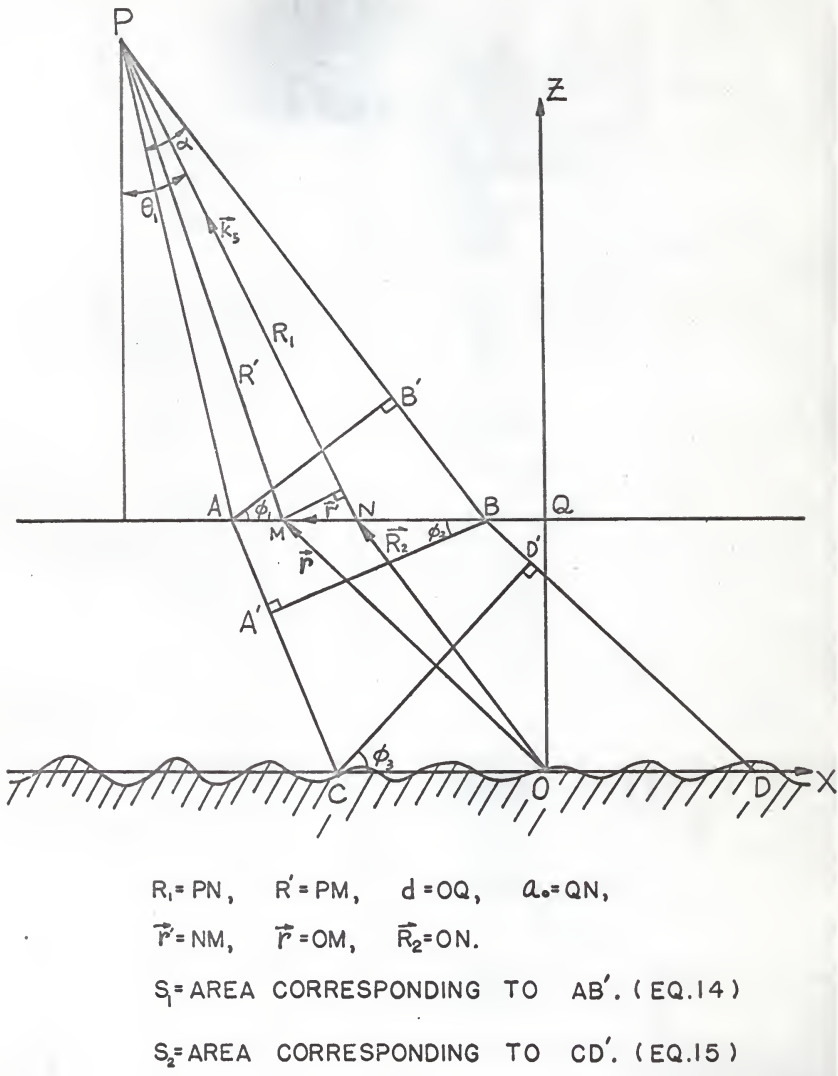

FIG.5. FURTHER DETAILS OF ROUGH LAYER SCATTER. 


$$
\begin{aligned}
E_{4} & =\frac{\cos \phi_{1}}{\cos \phi_{2}}\left[\frac{A B \cos \phi_{2}}{C D \cos \phi_{3}}\right] D_{12} E_{o} e^{j \vec{k}_{3} \cdot \vec{r}} \\
& =\frac{A B \cos \phi_{1}}{C D \cos \phi_{3}} \quad D_{12} E_{o} e^{j \vec{k}_{3} \cdot \vec{r}} \\
& =\frac{S_{1}}{S_{2}} D_{12} E_{\circ} e^{j \vec{k}_{3} \cdot \vec{r}}
\end{aligned}
$$

where

$$
\begin{aligned}
& S_{1}=A B \cos \phi_{1} . \\
& S_{2}=C D \cos \phi_{3} .
\end{aligned}
$$

$\vec{r}$ is given by (5), and angle $\phi_{3}$ is defined in Fig. 5 .

The beam with electric field $\mathrm{E}_{4}$ incident on the rough boundary will be scattered in all directions within the layer due to the roughness of the boundary. The scattering pattern will depend mainly upon the statistical properties of the rough boundary.

Within the layer, the mean backscattered power $\left\langle E_{5} E_{5}{ }^{*}\right\rangle$ at $\mathrm{AB}$ on the upper boundary, which is assumed to be in the Fraunhofer zone of diffraction or the far zone, is given by $(45 b)$ or $(63 b)$ of chapter 2 .

$$
\left\langle E_{5} E_{5}{ }^{*}\right\rangle=\left|E_{50}\right|^{2} \rho_{\mathrm{RMS}}{ }^{2}=\left|E_{50}\right|^{2}\langle\rho \rho *\rangle
$$

where $\mathrm{E}_{5}$ is the backscattered electric field at $\mathrm{AB}$ within the layer and $\mathrm{E}_{5}$ is its complex conjugate.

For one dimensionally rough surface, $\xi=\xi(x)$, the value of $\mathrm{E}_{50}$ is given by 
$E_{50}=j \frac{s_{1}}{S_{2}} \frac{D_{12} E_{0} k_{3} L \cos \theta_{2}}{\pi R_{2}} e^{j k_{3} R_{2}}$

and $\rho_{\text {RMS }}$ is given by (45), chapter 2 .

For the case of backscatter, which is being considered here, the values of $v_{x}, F_{2}, g$ and $\rho_{0}$ in (45), chapter 2 , are given by substituting the appropriate values $\left(\theta_{2}=-\theta_{1}, \theta_{1}=\theta_{2}\right.$ in that order; and $\left.\lambda=\lambda_{2}\right)$ in $(17 a),(27),(36),(44 a)$, chapter 2 . Then

$$
\begin{aligned}
& v_{X}=\frac{4 \pi}{\lambda_{2}} \sin \theta_{2} \\
& F_{2}=1 / \cos ^{2} \theta_{2} \\
& \sqrt{g}=\frac{4 \pi \sigma_{Z}}{\lambda_{2}} \cos \theta_{2} \\
& \rho_{O}=\frac{\sin v_{X} L}{v_{X} L} \\
& R_{2}=\text { ON (Fig. } 5 \text { ). } \\
& L=\text { OC }=\text { OD (Fig. 4). } \\
& T=\text { Correlation distance of rough surface } \\
& \quad \text { (lower boundary of layer). }
\end{aligned}
$$

For the two dimensional rough surface, $\xi=\xi(x, y)$, the value of $E_{50}$ is given by

$$
E_{50}=j \frac{S_{1}}{S_{2}} \frac{D_{12} E_{0} k_{3} A_{0} \cos \theta_{2} e^{j k_{3} R_{2}}}{2 \pi R_{2}}
$$

where $A_{0}$ is the area illuminated on the lower boundary of layer, and $\rho_{R M S}$ is given by $(63)$, chapter 2 . The values of $v_{x y}, F_{3}, g$ and $\rho_{0}$ in (63), chapter 2 , are given by substituting the appropriate 
values $\left(\theta_{2}=-\theta_{1}, \theta_{1}=\theta_{2}\right.$ in that order; $\theta_{3}=0$ and $\left.\lambda=\lambda_{2}\right)$ in $(58),(52 a),(36),(64)$, chapter 2 . Since the backscattered power is being calculated in the plane of incidence, the values of $v_{x y}$, $F_{3}, g, \rho_{0}$ and $R_{2}$ are given by (18), (19), (20), (21) and (22), respectively. $L$ and $T$ are defined by (23) and (24), respectively. The mean backscattered power $\left\langle\mathrm{E}_{5} \mathrm{E}_{5}{ }^{*}\right\rangle$ at $\mathrm{AB}$ within the layer is given by (16), and its square root will give the magnitude of the electric field at that point. It has been assumed that $A B$ is quite small and is situated in the Fraunhofer zone of diffraction. The backscattered electromagnetic wave over this region may therefore be assumed to be a plane wave given by

$$
<E_{5}>=\left|E_{50}\right| \rho_{R M S} e^{j \vec{k}_{4} \cdot \vec{r}}
$$

where $\rho_{\mathrm{RMS}}$ is given by either $(45)$ or $(63)$, chapter $2 ; \vec{r}$ is given by $(6)$, and

$$
\vec{k}_{4}=-\vec{k}_{3}
$$

is the propogation vector of the backscattered wave at $A B$.

The exact phase of the backscattered wave at $A B$ will vary from point to point. In (26) the phase has been approximated by its mean value $\exp \left(j \vec{k}_{4} \cdot \vec{r}\right)$. This may be done because $A B$ is quite small compared to $d$ and is in the far zone of diffraction. Then, the mean backscattered field $\left\langle E_{6}\right\rangle$ at $A B$ on the upper boundary of the layer, in medium 1 , will be given by

$$
\left\langle E_{6}\right\rangle=D_{21} \cdot\left|E_{50}\right| \rho_{R M S} e^{j \vec{k}_{5} \cdot \vec{r}}
$$

where $\rho_{\text {RMS }}$ is given by either $(45 a)$ or $(63 a)$, chapter $2 ; \vec{r}$ is given by $(6)$; 
$\overrightarrow{\mathrm{k}}_{5}=-\overrightarrow{\mathrm{k}}_{1}$

is the propogation vector of the backscattered wave in medium 1 , and

$$
\begin{aligned}
\mathrm{D}_{21} & =1+\mathrm{v}_{21} \\
& =1-\mathrm{v}_{12}
\end{aligned}
$$

is the transmission coefficient at the upper boundary for a wave travelling from medium 2 into medium. 1.

\subsection{Radar cross section of rough layer}

The radar cross section $\sigma$ of a target is defined as the area intercepting that amount of power which, when scattered equally in all directions, produces an echo at the radar equal to that from the target. Mathematically,

$$
\begin{aligned}
& \sigma=\frac{\text { Power reflected towards radar receiver/unit solid angle }}{\text { incident power density on upper boundary of layer } / 4 \pi} \\
& =\lim _{R \rightarrow \infty} \underset{\pi R^{2}}{4}\left|\frac{E_{r}}{E_{i}}\right| \text {, }
\end{aligned}
$$

where

$$
\begin{aligned}
& R=\text { distance between radar and target, } \\
& E_{r}=\text { reflected electric field strength at radar receiver, } \\
& E_{i}=\text { strength of electric field incident on target. }
\end{aligned}
$$

For the layer, the mean value of radar cross section is given by

$$
\langle\sigma\rangle=\lim _{R_{1} \rightarrow \infty} 4 \pi R_{1}{ }^{2} \cdot\left|\frac{\langle E(P)\rangle}{E_{1}}\right|^{2},
$$


where

$$
\begin{aligned}
R_{1}=P N & (\text { Fig. } 5) . \\
\langle E(P)>= & \text { Mean value of the backscattered electric field at } \\
& \text { the radar receiver. }
\end{aligned}
$$

It is more convenient to calculate the mean value of $\sigma_{0}$ ' the normalized radar cross section (or differential cross section) of the target, which is defined as

$$
\begin{aligned}
& \sigma_{0}=\frac{\sigma}{S_{0}} \\
& =\lim _{R_{1} \rightarrow \infty} \frac{4 \pi R_{1}^{2}}{S_{0}}\left|\frac{E(P)}{E_{1}}\right|^{2}
\end{aligned}
$$

where $S_{0}$ is the area of the illuminated surface $A B$, on the upper boundary of layer.

In (33) the only unknown quantity is $E(P)$. Before $E(P)$ is evaluated, the incident wave $\left(E_{1}\right)$ will be redefined so as to be in conformity with actual practice. Instead of the incident wave being considered a continuous wave, henceforward it shall be assumed to consist of a pulse. If the pulse is long enough, then it is a good approximation to a continuous plane harmonic wave while it lasts. Pulsed incidence is of two types: (1) Beamwidth limited (2) Pulse-width limited. In what follows, $E_{1}$ shall be assumed to consist of a pulse of the beam-width limited type. It will also be assumed that there is no elongation of the reflected or scattered pulse.

The length of the pulse is taken as $T_{0}$ seconds, and the time taken by the pulse front to travel from $A B$ at the upper 
boundary of layer to the lower boundary and back again to the upper boundary as $2 t_{0}$ seconds. It is assumed that $t=0$, when the pulse front passes through $N$, the middle point of $A B$.

To evaluate $E(P)$, the Helmholtz integral defined by (5), chapter 2 will be used. In this integral $E$ and $\frac{\partial E}{\partial n}$ are the electric field and its normal derivative on $A B$ (or surface $s_{0}$ ) at the upper boundary of layer in medium 1. The value of (E) $\mathrm{S}_{0}$ and $\left(\frac{\partial E}{\partial n}\right)_{S_{0}}$ will vary with time, having different values in different time intervals. These values will depend upon the pulse repetition frequency (PRF) and the value of $T_{0}$ compared with $2 t_{0}$. It is assumed that the PRF chosen is such that no two pulses interfere with each other. i.e., the time interval between two pulses is greater than the total time taken for one pulse to travel from the radar to the layer and back again. Two possibilities for the comparative values of $T_{0}$ and $2 t_{0}$ are:

(1) $T_{0}<2 t_{0}$, and

(2) $\mathrm{T}_{0}>2 t_{0}$.

When $T_{0}<2 t_{0}$, the pulse shall be denoted "short pulse" and the values of $E(P)$ and $\sigma_{0}$ calculated for each time interval shall be grouped under Case I. Similarly, when $T_{0}>2 t_{0}$, the pulse shall be denoted "long pulse" and the values of $E(P)$ and $\sigma_{0}$ calculated for each time interval shall be grouped under Case II.

Case I: Short pulse $\left(T_{0}<2 t_{0}\right)$.

For each pulse the values of $(E) S_{\circ}$ and $\left(\frac{\partial E}{\partial n}\right)_{S_{0}}$ can be grouped into three different time intervals. These values, will of course 
be repetitive for every pulse.

(1) Time interval: $0<t<T_{0}$

During this time interval, the total electric field on $A B$ will be given by the sum of (3) and (9), which represents the incident and reflected waves, respectively:

${ }^{(E)} S_{0}=\left[E_{1}+E_{2}\right]_{S_{0}}$

$$
=\left[E_{0} e^{j \vec{k}_{1} \cdot \vec{r}}+v_{12} E_{0} e^{j \vec{k}_{2} \cdot \vec{r}}\right]_{S_{0}}
$$

The normal derivative of $E$ on $A B$ is given by

$$
\begin{aligned}
& \left(\frac{\partial E}{\partial n}\right)_{S_{O}}=(\nabla E \cdot \vec{n}) S_{O}
\end{aligned}
$$

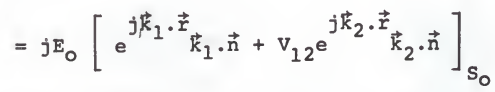

$$
\begin{aligned}
& =j E_{0}\left[e^{j \vec{k}_{1} \cdot \vec{r}}-v_{12} e^{j \vec{k}_{2} \cdot \vec{r}}\right] s_{0} \vec{k}_{1} \cdot \vec{n}
\end{aligned}
$$

since

$$
-\vec{k}_{1} \cdot \vec{n}=+\vec{k}_{2} \cdot \vec{n}=+\vec{k}_{5} \cdot \vec{n}
$$

Substituting $k_{2}=k_{5}$ in $(6)$, chapter 2 , gives

$$
\psi=\frac{e^{j}}{R^{\prime}}
$$

where $R^{\prime}=\operatorname{PM}$ (Fig. 5).

From Fig. 5, it will be seen that

$$
k_{5} R^{\prime}=k_{5} R_{1}-\vec{k}_{5} \cdot \vec{r}^{\prime}
$$


where

$$
\vec{r}^{\prime}=\vec{r}-\vec{R}_{2}
$$

Therefore

$$
\begin{aligned}
k_{5} R^{\prime} & =k_{5} R_{1}-\vec{k}_{5} \cdot \vec{r}+\vec{k}_{5} \cdot \vec{R}_{2} \\
& =k_{5}\left[R_{1}+R_{2} \cos \left(\theta_{1}-\theta_{2}\right)\right]-\vec{k}_{5} \cdot \vec{r}
\end{aligned}
$$

and (37) reduces to

$$
(\Psi)_{S_{0}}=\left[\frac{e^{j k_{5}\left[R_{1}+R_{2} \cos \left(\theta_{1}-\theta_{2}\right)\right]-j \vec{k}_{5} \cdot \vec{r}}}{R_{1}}\right]_{S_{0}}
$$

where the substitution $R_{1} \doteq R^{\prime}$ has been made in the denominator. This is justified, since the point $P$ is in the far zone. The normal derivative of $\psi$ on $A B$ is given by

$$
\left(\frac{\partial \psi}{\partial n}\right)_{S_{0}}=(\nabla \psi \cdot \vec{n})_{S_{0}}=\left[-j \psi \vec{k}_{5} \cdot \vec{n}\right] S_{0}
$$

Substituting (34), (35), (36), (39), (40) in (5), chapter 2, and simplifying we get

$E(P)=\frac{j E_{0} v_{12} e^{j k_{5}\left[R_{1}+R_{2} \cos \left(\theta_{1}-\theta_{2}\right)\right]}\left(\vec{k}_{1} \cdot \vec{n}\right)}{2 \pi R_{1}} \iint_{s_{0}} e^{j\left(\vec{k}_{2}-\vec{k}_{5}\right) \cdot \vec{r}} d s$

where

$$
\begin{aligned}
& \vec{k}_{1} \cdot \vec{n}=-k_{1} \cos \theta_{1} \\
& \vec{k}_{2}=\frac{2 \pi}{\lambda_{1}}\left(\sin \theta_{1} \cdot \vec{a}_{x}+\cos \theta_{1} \cdot \vec{a}_{z}\right)
\end{aligned}
$$




$$
\vec{k}_{5}=\frac{2 \pi}{\lambda_{1}}\left(-\sin \theta_{1} \vec{a}_{x}+\cos \theta_{1} \vec{a}_{z}\right) \quad
$$

Since the upper boundary is smooth $\theta_{1}{ }^{\prime}=\theta_{1}$ and (43) reduces to

$$
\vec{k}_{2}=\frac{2 \pi}{\lambda_{1}}\left(\sin \theta_{1} \vec{a}_{x}+\cos \theta_{1} \vec{a}_{z}\right) \text {. }
$$

Substituting (42), (44), (44a) in (41) and setting the limits of integration, we get

$$
\langle E(P)\rangle=\frac{(-j) k_{1} E_{0} V_{12} \cos \theta_{1} e^{j k_{5}\left[R_{1}+R_{2} \cos \left(\theta_{1}-\theta_{2}\right)\right]}}{2 \pi R_{1}} \cdot I_{1}
$$

where

$$
\begin{aligned}
& I_{1}=\int_{-X_{0}-a_{0}}^{+X_{0}-a_{0}} \int_{-Y_{0}}^{+Y_{0}} e^{\frac{j 4 \pi}{\lambda} \sin \theta_{1} x} d x d y=s_{0} e^{\frac{j 4 \pi}{\lambda_{1}} a_{0} \sin \theta_{1}} \frac{\sin \phi}{\phi} \\
& \phi=\frac{4 \pi}{\lambda_{1}} x_{0} \sin \theta_{1} \\
& S_{0}=4 X_{0} Y_{0} \\
& a_{0}=Q N(\text { Fig. 5). }
\end{aligned}
$$

Substituting (4) and (46) in (45), we get

$\langle E(P)\rangle=\frac{(-j) E_{0} V_{12} S_{0} \cos \theta_{1} e^{j \frac{2 \pi}{\lambda_{1}}\left[R_{1}+R_{2} \cos \left(\theta_{1}-\theta_{2}\right)-2 a_{0} \sin \theta_{1}\right]}}{\lambda_{1} R_{1}} \cdot \frac{\sin \phi}{\phi}$

This value of $\langle E(P)\rangle$ is substituted in (33) to give the mean value of the normalized radar cross section of the layer for the short pulse case during the time interval $0<t<\mathrm{T}_{0}$ : 
$\left\langle\sigma_{0}\right\rangle=\frac{4 \pi v_{12}{ }^{2} s_{0} \cos ^{2} \theta_{1}}{\lambda_{1}{ }^{2}}\left|\frac{\sin \phi}{\phi}\right|^{2}$

In the above equation as $\frac{\lambda_{1}}{x_{0}} \rightarrow 0, \frac{\sin \phi}{\phi} \rightarrow 1$ for normal incidence and $\frac{\sin \phi}{\phi} \rightarrow 0$ for all other angles of incidence. Therefore it may be concluded that, except in case of normal incidence, the value of $\left\langle\sigma_{0}\right\rangle$ will be very small in all other directions.

(2) Time interval: $T_{0}<t<2 t_{0}$

In this time interval, the electric field on $A B$ is zero, since the field due to the wave scattered by the lower boundary of the layer is not present:

$$
{ }^{(E)} S_{0}=0
$$

and therefore during this time interval

$$
\left\langle\sigma_{0}\right\rangle=0
$$

(3) Time interval: $2 t_{0}<t<2 t_{0}+T_{0}$

In this time interval the electric field present on $A B$ is due to the wave scattered by the lower boundary of layer, and is given by (28)

${ }^{(E)_{S_{0}}}=\left[\left\langle E_{6}\right\rangle\right]_{S_{0}}=\left[D_{21}\left|E_{50}\right| \rho_{R M S} e^{j \vec{k}_{5} \cdot \vec{r}}\right]_{S_{0}}$.

The normal derivative is given by

$$
\left(\frac{\partial E}{\partial n}\right)_{S_{0}}(\nabla E \cdot \vec{n}) S_{0}=\left[j D_{21}\left|E_{50}\right| \rho_{R M S} e^{\left.j \vec{k}_{5} \cdot \vec{r}_{k_{5}} \cdot \vec{n}\right]} S_{\circ}\right.
$$

Substituting (39), (40), (54), (55) in (5) Chapter 2, setting limits of integration and simplifying, one gets 
$\langle E(P)\rangle=\frac{(-j) D_{21}\left|E_{50}\right| \rho R M S}{e^{j k_{5}\left[R_{1}+R_{2} \cos \left(\theta_{1}-\theta_{2}\right)\right]}} \cdot I_{2}$

where

$$
I_{2}=\int_{-x_{0}-a_{0}}^{+x_{0}-a_{0}} \int_{-y_{0}}^{+y_{0}}\left(\vec{k}_{5} \cdot \vec{n}\right) d x d y
$$

Substituting (4), (36), (42), (56a) in (56), and solving the integral, the result is

$$
\langle E(P)\rangle=\frac{(-j) D_{21}\left|E_{50}\right| \rho_{R M S} s_{0} \cos \theta_{1} e^{j \frac{2 \pi}{\lambda_{1}}\left[R_{1}+R_{2} \cos \left(\theta_{1}-\theta_{2}\right)\right]}}{\lambda_{1} R_{1}} .
$$

The above equation is substituted in (33), to give the mean value of the normalized radar cross section of the layer for the short pulse case during the time interval $2 t_{0}<t<2 t_{0}+T_{0}$ :

$$
<\sigma_{\rho}>=\frac{4 \pi \mathrm{D}_{21}{ }^{2}\left|\mathrm{E}_{50}\right|^{2} \rho_{\mathrm{RMS}}{ }^{2} \mathrm{~S}_{\mathrm{O}} \cos ^{2} \theta_{1}}{\lambda_{1}{ }^{2} \mathrm{E}_{\mathrm{O}}{ }^{2}}
$$

The statistical properties of the rough boundary of layer are contained in ${ }^{\mathrm{R}} \mathrm{RS}$, and $\left|\mathrm{E}_{50}\right|$ is a function of the mean depth of layer $d$, the incident field strength $\mathrm{E}_{\mathrm{O}}$, etc., and is given by (17) and (25) for the case of one and two dimensional rough surface, respectively.

Case II: Long Pulse $\left(\mathrm{T}_{\mathrm{O}}>2 \mathrm{t}_{0}\right)$.

In this case also the values of ${ }^{(E)_{S_{O}}}$ and $\left(\frac{\partial E}{\partial n}\right)_{S_{O}}$ for each pulse can be grouped into three different time intervals. These 
values will be repetitive for every pulse.

(1) Time interval: $0<t<2 t_{0}$

In this time interval (E) $\mathrm{S}_{\mathrm{O}}$ is given by (34) and consequently $\left\langle\sigma_{0}\right\rangle$, the mean value of the normalized radar cross section of layer is given by (51).

(2) Time interval: $2 t_{0}<t<T_{0}$

In this time interval the total electric field on $A B$ will be given by the sum of (3), (9) and (28), which represent the sum of the incident wave, the reflected wave and the wave scattered by the lower boundary of layer, respectively.

$$
\begin{aligned}
{\text { (E) } S_{0}}=\left[E_{1}+E_{2}+\left\langle E_{6}\right\rangle\right] S_{0} \\
=\left[E_{O} e^{j \vec{k}_{1} \cdot \vec{r}}+V_{12} E_{0} e^{j \vec{k}_{2} \cdot \vec{r}}+D_{12} E_{0} \rho_{R M S} e^{j \vec{k}_{5} \cdot \vec{r}}\right] S_{0}
\end{aligned}
$$

Since (59) is a sum of (34) and (54), therefore, for this time interval, $\langle E(P)>$ will be given by the sum of $(50)$ and (57)

$$
\begin{aligned}
& \langle E(P)\rangle=\frac{(-j) E_{0} V_{12} S_{0} \cos \theta_{1} e^{j \frac{2 \pi}{\lambda_{1}}\left[R_{1}+R_{2} \cos \left(\theta_{1}-\theta_{2}\right)-2 a_{0} \sin \theta_{1}\right]}}{\lambda_{1} R_{1}} \cdot \frac{\sin \phi}{\phi} \\
& +\frac{(-j) D_{21}\left|E_{50}\right| \rho_{R M S} S_{0} \cos \theta_{1} e^{j \frac{2 \pi}{\lambda_{1}}\left[R_{1}+R_{2} \cos \left(\theta_{1}-\theta_{2}\right)\right]}}{\lambda_{1} R_{1}} \\
& =\frac{(-j) s_{0} \cos \theta_{1} e^{j \frac{2 \pi}{\lambda_{1}}\left[R_{1}+R_{2} \cos \left(\theta_{1}-\theta_{2}\right)\right]}}{\lambda_{1} R_{1}}\left[E_{0} v_{12} e^{j \frac{4 \pi}{\lambda_{1}} a_{0} \sin \theta_{1}} \frac{\sin \phi}{\phi}\right. \\
& \left.+D_{21}\left|E_{50}\right| \rho_{R M S}\right] \text {. }
\end{aligned}
$$


Substituting $(60)$ in (33) gives the mean value of the normalized radar cross section of the layer for the long pulse case during the time interval $2 t_{0}<t<T_{0}:$

$\left\langle\sigma_{0}\right\rangle=\frac{4 \pi s_{0} \cos ^{2} \theta_{1}}{\lambda_{1}{ }^{2}}\left|v_{12} e^{j \frac{4 \pi}{\lambda_{1}} a_{0} \sin \theta^{1_{1} \sin \phi}} \frac{D_{21}\left|E_{50}\right| \rho_{R M S}}{E_{0}}\right|^{2}$

As discussed earlier, $\frac{\sin \phi}{\phi} \rightarrow 1$ for normal incidence and $\frac{\sin \phi}{\phi} \rightarrow 0$ for all other angles of incidence. Therefore, except in case of normal incidence, the second term of (61) will be much greater than the first term. Again $\rho_{\text {RMS }}$ contains the statistics of the rough boundary and $\left|E_{50}\right|$ is a function of $d, E_{0}$, etc., given by (17) and (25) for the case of one and two dimensional rough surface, respectively.

(3) Time interval: $T_{0}<t<T_{0}+2 t_{0}$

In this time interval $\left(E_{S_{0}}\right.$ is given by $(28)$ and subsequently $\left\langle\sigma_{0}\right\rangle$, the mean value of the normalized radar cross section of layer, is given by (58).

\subsection{Summary of Results}

Case I: Short Pulse $\mathrm{T}_{\mathrm{o}}<2 \mathrm{t}_{\mathrm{O}}$

In this case the pulse duration is less than the time of a two-way trip within the layer.

(1) Time interval: $0<t<T_{0}$

$$
\left\langle\sigma_{0}\right\rangle=\frac{4 \pi \mathrm{v}_{12}{ }^{2} \mathrm{~s}_{0} \cos ^{2} \theta_{1}}{\lambda_{1}{ }^{2}}\left|\frac{\sin \phi}{\phi}\right|^{2} \text {. }
$$


Layer appears only as a smooth plane.

(2) Time interval: $T_{0}<t<2 t_{0}$

$\left\langle\sigma_{0}\right\rangle=0$

No backscattered signal from layer.

(3) Time interval: $2 t_{0}<t<2 t_{0}+T_{0}$

$$
<\sigma_{0}>=\frac{4 \pi \mathrm{D}_{21}{ }^{2}\left|\mathrm{E}_{50}\right|^{2} \rho^{2} \mathrm{RMS}_{\mathrm{o}} \cos ^{2} \theta_{1}}{\lambda_{1}{ }^{2} \mathrm{E}_{\mathrm{o}}{ }^{2}}
$$

Layer appears only as a rough surface.

\section{Case II: Long Pulse $\left(\mathrm{T}_{0}>2 \mathrm{t}_{0}\right)$}

In this case the pulse duration is larger than the time of a two-way trip within the layer.

(1) Time interval: $0<t<2 t_{0}$

$$
\left\langle\sigma_{0}>=\frac{4 \pi v_{12}{ }^{2} s_{0} \cos ^{2} \theta_{1}}{\lambda_{1}{ }^{2}}\left|\frac{\sin \phi}{\phi}\right|^{2}\right.
$$

Layer appears only as a smooth plane.

(2) Time interval: $2 t_{0}<t<T_{0}$

$\left\langle\sigma_{0}\right\rangle=\frac{4 \pi S_{0} \cos ^{2} \theta_{1}}{\lambda_{1}{ }^{2}} \mid\left(\left.V_{12} e^{j \frac{4 \pi a_{0} \sin \lambda_{1}}{\lambda_{1}}} \frac{\sin \phi}{\phi} \frac{+D_{21}\left|E_{50}\right| \rho_{R M S}}{E_{0}}\right|^{2}\right.$

Layer appears smooth at normal incidence and rough as angle of incidence departs from normal.

(3) Time interval: $T_{0}<t<T_{0}+2 t_{0}$ 


$$
\left\langle\sigma_{0}\right\rangle=\frac{4 \pi D_{21}{ }^{2}\left|E_{50}\right|^{2} \rho_{\text {RMS }}{ }^{2} S_{0} \cos ^{2} \theta_{1}}{\lambda_{1}{ }^{2} E_{0}{ }^{2}} .
$$

Layer appears only as a rough surface.

For both Cases I \& II, the reflection coefficient $V_{12}$ is given in Eq. (11) or (12), chapter 2 and transmission coefficient $D_{21}$ is given in Eq. (30); also the RMS backscattering coefficient PRMS is given by Eq. (45) or (63), chapter 2 .

\subsection{Discussion of Results}

Case I: Short Pulse $\left(T_{0}<2 t_{0}\right)$

Time interval: $0<t<T_{0}$

In this time interval, the backscattered signal at the radar receiver is the signal reflected by the smooth front surface of the layer only, and the value of $\left\langle\sigma_{0}\right\rangle$, the mean normalized radar cross section of layer, is given by $(62)$. In this equation < $\sigma_{0}$ > is proportional to $\frac{\sin \phi}{\phi}$, where

$$
\phi=\frac{4 \pi}{\lambda_{1}} x_{0} \sin \theta_{1}
$$

$\theta_{1}=$ angle of incidence at front surface (Fig. 4).

$\lambda_{1}=$ wavelength in medium 1.

$x_{0}=$ Beamwidth (in the direction of the $x$-coordinate) of the incident wave at the front surface.

In $(62)$, as $\frac{\lambda_{1}}{x_{0}} \rightarrow 0$, the quantity

$\frac{\sin \phi}{\phi}+1$ for normal incidence, and

$\underline{\sin \phi} \rightarrow 0$ as the angle of incidence departs from normal. 
Hence $\left\langle\sigma_{0}\right\rangle \rightarrow$ a certain maximum value for normal incidence and $<\sigma_{0}>+0$ as the angle of incidence departs from normal. This should be expected from a smooth surface.

As a check, the value of $\left\langle\sigma_{0}\right\rangle$ is evaluated for normal incidence. Substituting $\frac{\sin \phi}{\phi}=1$ and $\cos ^{2} \theta_{1}=1$ in $(62)$, gives $\left\langle\sigma_{0}\right\rangle=4 \pi \frac{v_{12}{ }^{2}}{\lambda_{1}{ }^{2}} S_{0}$.

For a perfect conductor $v_{12}=1$, and $(68)$ reduces to

$\left\langle\sigma_{0}\right\rangle=4 \pi \frac{s_{0}}{\lambda_{1}{ }^{2}}$

which is the well known result for the radar cross section of a flat metal plate of area $s_{0}[33,36]$.

Time interval: $2 t_{0}<t<2 t_{0}+T_{0}$

In this time interval, the backscattered signal at the radar receiver is from the rough back surface of the layer only, and the value of $\left\langle\sigma_{0}\right\rangle$ is given by $(64)$.

(a) Dependence on $\theta_{1}$ : In $(64), \sigma_{0}$ is proportional to $\cos ^{2} \theta_{1}$, where $\theta_{1}$ is the angle of incidence at the upper boundary of layer. Therefore, as $\theta_{1} \rightarrow 0, \cos ^{2} \theta_{1} \rightarrow 1$ and $\left\langle\sigma_{0}\right\rangle+$ a certain maximum value. Again as $\theta_{1}+\frac{\pi}{2}, \cos ^{2} \theta_{1}>0$ and $\left\langle\sigma_{0}\right\rangle+0$; this should be expected because $\theta_{1}+\frac{\pi}{2}$ is the case of grazing incidence (at the upper smooth boundary), when $\mathrm{v}_{12}+1$ and the incident wave will be fully reflected at the upper boundary, no energy being transmitted into the layer. 
(b) Dependence on $\rho_{\mathrm{RMS}}$ : In $(64),\left\langle\sigma_{0}\right\rangle$ is also proportional to $\rho^{2}$ RMS, which is a function of $g$, where

$\sqrt{ } g=4 \pi \frac{\sigma_{z}}{\lambda_{2}} \cos \theta_{2}$.

$\sigma_{z}=$ standard deviation of the heights of the rough surface (back surface) of layer.

$\lambda_{2}=$ wavelength within the layer (in medium 2).

$\theta_{2}=$ angle of refraction at upper boundary of layer. To analyse the dependence of $\left\langle\sigma_{0}\right\rangle$ on $\rho_{\mathrm{RMS}}$, two cases will be considered:

(a) $g \ll 1$, and

(b) $\mathrm{g} \gg 1$.

Since $/ g$ is proportional to $\sigma_{z} / \lambda_{2}$, these two cases correspond to a slightly rough and a very rough surface.

The mean square scatter coefficient for the two cases, as given by Beckmann $[5$, p. 88], is shown below.

For a surface rough in only one dimension

$$
\begin{aligned}
& \rho_{\text {RMS }}^{2}=\left\langle\rho \rho^{*}\right\rangle=e^{-g} \rho_{O}{ }^{2}+\frac{\sqrt{ } F_{2}{ }^{2} \mathrm{Tg}}{2 \mathrm{~L}} e^{-V_{X}{ }^{2} \mathrm{~T}^{2} / 4} \quad g \ll 1 \\
& \rho_{\text {RMS }}^{2}=\left\langle\rho \rho *=\frac{\mathrm{TF}_{2}{ }^{2} / \pi}{2 \mathrm{Lv}_{\mathrm{z}} \sigma_{\mathrm{z}}} \exp \cdot\left(-\frac{\mathrm{v}_{\mathrm{x}}{ }^{2} \mathrm{~T}^{2}}{4 \mathrm{v}_{\mathrm{z}}{ }^{2} \sigma_{\mathrm{z}}{ }^{2}}\right) \quad g>1\right.
\end{aligned}
$$

For a surface rough in both dimensions

$$
\rho_{\mathrm{RMS}}^{2}=\mathrm{e}^{-\mathrm{g}}\left(\rho_{\mathrm{O}}{ }^{2}+\frac{\pi \mathrm{T}^{2} \mathrm{~F}_{3}{ }^{2} \mathrm{~g}}{\mathrm{~A}_{\mathrm{O}}} \mathrm{e}^{-\mathrm{v}_{\mathrm{XY}}{ }^{2} \mathrm{~T}^{2} / 4}\right) \quad \mathrm{g} \ll 1
$$


$\rho_{\text {RMS }}^{2}=\frac{\pi F_{3}{ }^{2} \mathrm{~T}^{2}}{\mathrm{~A}_{0} \mathrm{v}_{\mathrm{z}}{ }^{2} \sigma_{\mathrm{z}}{ }^{2}} \exp \cdot\left(-\frac{\mathrm{v}_{\mathrm{xy}}{ }^{2} \mathrm{~T}^{2}}{4 \mathrm{v}_{\mathrm{z}}{ }^{2} \sigma_{\mathrm{z}}{ }^{2}}\right) \quad g \gg 1$

For $g \ll 1$ (nearly smooth surface), it can be seen from (70) and (72) that the specular term $\left(e^{-g_{\rho_{0}}}{ }^{2}\right)$ is the dominant term, since $T \ll I$ or $T^{2} \ll A$. Therefore, in $(64), \ll \sigma_{0}>$ will have a significant value only for normal incidence, and will decrease as the angle of incidence departs from normal. For $g \gg 1$ (very rough surface), (71) and (73) show that the specular term $\left(e^{\left.-g_{\rho_{0}}{ }^{2}\right)}\right.$ is no longer present and the dependence on the scattering angle $\left(\theta_{2}, \theta_{3}^{-}, \mathrm{Fig}, 1, \mathrm{Ch} .2\right)$ is reduced to that contained in $\mathrm{F}_{2}$ or $\mathrm{F}_{3}, \mathrm{v}_{\mathrm{x}}$ or $v_{x y}$, and $g$. This dependence of $\rho^{2}$ RMS on $g$ (i.e., on the surface roughness $\frac{\sigma_{z}}{\lambda}$ ), shows that as $g$ increases or in other words the surface becomes more rough, $\rho^{2}$ RMS decreases and the scattering becomes diffuse. Therefore as $g$ increases, the value of $\left\langle\sigma_{0}>\right.$ in (64) will decrease.

\section{Case II: Long Pulse $\mathrm{T}_{\mathrm{O}}>2 \mathrm{t}_{\mathrm{O}}$}

The above discussion for Case I (Short Pulse) also holds for Case II (Long Pulse), since the value of < $\left.\sigma_{0}\right\rangle$ for this case is given by $(65),(66),(67)$, and these expressions are similar to the corresponding Case I expressions (62) and (64).

It should be pointed out that the results of this chapter are an extension of Beckmann's work [5]. The essential difference is that in case of Beckmann, the backscattered signal from only one surface (rough) is considered, whereas in the case of a 
rough layer, the backscattered signal from two interfaces (one smooth and one rough) is considered.

\subsection{Notation}

The notation for this chapter is collected here for convenience.

$\xi$ = function representing rough (lower) boundary of layer.

$\mathrm{S}_{\circ}=$ area $\mathrm{AB}=$ area illuminated on upper boundary of layer.

$A_{O}=$ area $C D=$ area illuminated on lower boundary of layer.

$L=O C=O D$ (Fig. 5).

$a_{0}=Q N($ Fig. 5).

$\vec{r}^{\prime}=$ vector NM (Fig. 5).

$I$ = radius vector $(\mathrm{Fig} .5)$.

$\vec{k}_{2}=$ vector ON (Fig. 5).

$d=$ mean depth of layer .

$\mathrm{R}_{1}=\mathrm{PN}=$ distance from radar of middle point of area illuminated

on upper boundary of layer (Fig. 5).

$\mathrm{R}_{2}=\mathrm{ON}=$ distance from origin of middle point of area illuminated on upper boundary of layer (Fig. 5).

$\mathrm{R}^{\prime}=\mathrm{PM}=$ distance from radar of any general point on the area illuminated on upper boundary of layer (Fig. 5).

$\phi_{1}=$ angle $B^{\prime} A B$ (Fig. 5).

$\phi_{2}=$ angle $\mathrm{ABA}$ ' (Fig. 5).

$\phi_{3}=$ angle $D^{\prime} C D$ (Fig. 5).

$\theta_{1}=$ angle of incidence at upper boundary (Fig. 4).

$\theta_{1}^{\prime}=$ angle of reflection at upper boundary (Fig. 4).

${ }^{\theta_{2}}=$ angle of refraction at upper boundary (Fig. 4). 
$\varepsilon_{1}, \mu_{1}=$ electromagnetic constants of medium 1 (Fig. 4).

$\varepsilon_{2}, \mu_{2}=$ electromagnetic constants of medium 2 (Fig. 4).

$\sigma_{3}=\infty=$ conductivity of medium 3 (Fig. 4).

$v_{12}=$ the reflection coefficient at the upper boundary between media 1 and 2 for a wave travelling from medium 1 into medium 2 .

$D_{12}=$ the transmission coefficient at the upper boundary between media 1 and 2 for a wave travelling from medium 1 into medium 2 .

$\mathrm{v}_{21}=$ the reflection coefficient at the upper boundary between media 1 and 2 for a wave travelling from medium 2 into medium 1 .

$D_{21}=$ the transmission coefficient at the upper boundary between media 1 and 2 for a wave travelling from medium 2 into medium 1.

$\lambda_{1}=$ wavelength in medium 1.

$\lambda_{2}=$ wavelength in medium 2.

$E_{1}=$ incident electric field on upper boundary of layer.

$E_{0}=\left|E_{1}\right|=$ magnitude of electric field incident on upper boundary of layer.

$\mathrm{E}_{2}=$ reflected electric field on upper boundary of layer.

$E_{3}=$ refracted electric field, just below the upper boundary of layer.

$E_{4}=$ electric field incident on lower boundary (rough surface) of layer.

$E_{5}=$ backscattered electric field at upper boundary, within the layer. 
$\mathrm{E}_{5}{ }^{*}=$ complex conjugate of $\mathrm{E}_{5}$.

$E_{50}=$ defined by equations (17) and (25), Chapter 3.

$\mathbf{E}_{6}=$ backscattered electric field at upper boundary, in medium 1.

$\langle E(P)\rangle=$ mean value of backscattered electric field at radar receiver.

$\vec{k}_{1}=$ propagation vector of wave incident on upper boundary of layer $\vec{k}_{2}=$ propagation vector of wave reflected by upper boundary of layer $\vec{k}_{3}=$ propagation vector of wave incident on lower boundary of layer $\vec{k}_{4}$ = propagation vector of the backscattered wave, within the layer $\vec{k}_{5}=$ propagation vector of the backscattered wave, in medium 1 . $\rho_{\mathrm{RMS}}=$ mean square backscatter coefficient of lower boundary of layer, given by equations (45) and (63), Chapter 2 .

$\sigma_{z}=$ the standard deviation of the heights of the rough boundary of layer.

$\mathrm{T}$ = correlation distance of rough boundary of layer.

$\sigma=$ radar cross section of layer.

$\sigma_{0}=$ normalized radar cross section of layer $=\sigma /$ area of layer surface.

$\left\langle\sigma_{0}\right\rangle=$ average value of $\sigma_{0}$

$T_{0}=$ incident pulse length.

$2 t_{0}=$ total time taken by the pulse front to travel from upper boundary of layer to lower boundary and back again to upper boundary.

$\mathrm{S}_{1}=$ cross-section of beam incident on upper boundary of layer.

$s_{2}=$ cross-section of beam incident on lower boundary of layer. 
4. RADAR CROSS SECTION MEASUREMENTS FOR A ROUGH LAYER BY ACOUSTIC SIMULATION TECHNIQUES.

An experimental study of the behaviour of a rough layer was undertaken to obtain some direct measurements of its average radar cross section. Acoustic simulation techniques were used for this study.

\subsection{Basic Concepts.}

The basis for acoustic simulation is the well known analogy between acoustic and electromagnetic waves. By considering the pressure or particle velocity of acoustic waves as the analog of the electric field intensity in the electromagnetic wave, one can obtain analogous expressions for parameters of interest in the two cases (e.g., impedance, reflection coefficient, velocity of propagation, etc.) Although polarization effects cannot be accoustically simulated due to the fact that acoustic propagation is a scalar phenomenon whereas electromagnetic waves are in general described by vectors, the phenomena of propagation, reflection, refraction and scatter that are of interest in radar can be studied in the laboratory by means of an acoustic simulator. This simulation is made more convenient and economical by the proper choice of frequency and medium of propagation; ultrasonic waves in water are used. It can easily be shown that quantitative information (rather than merely qualitative analogy) can be gained by suitable scaling of frequency, range, impedance, or other parameters. 


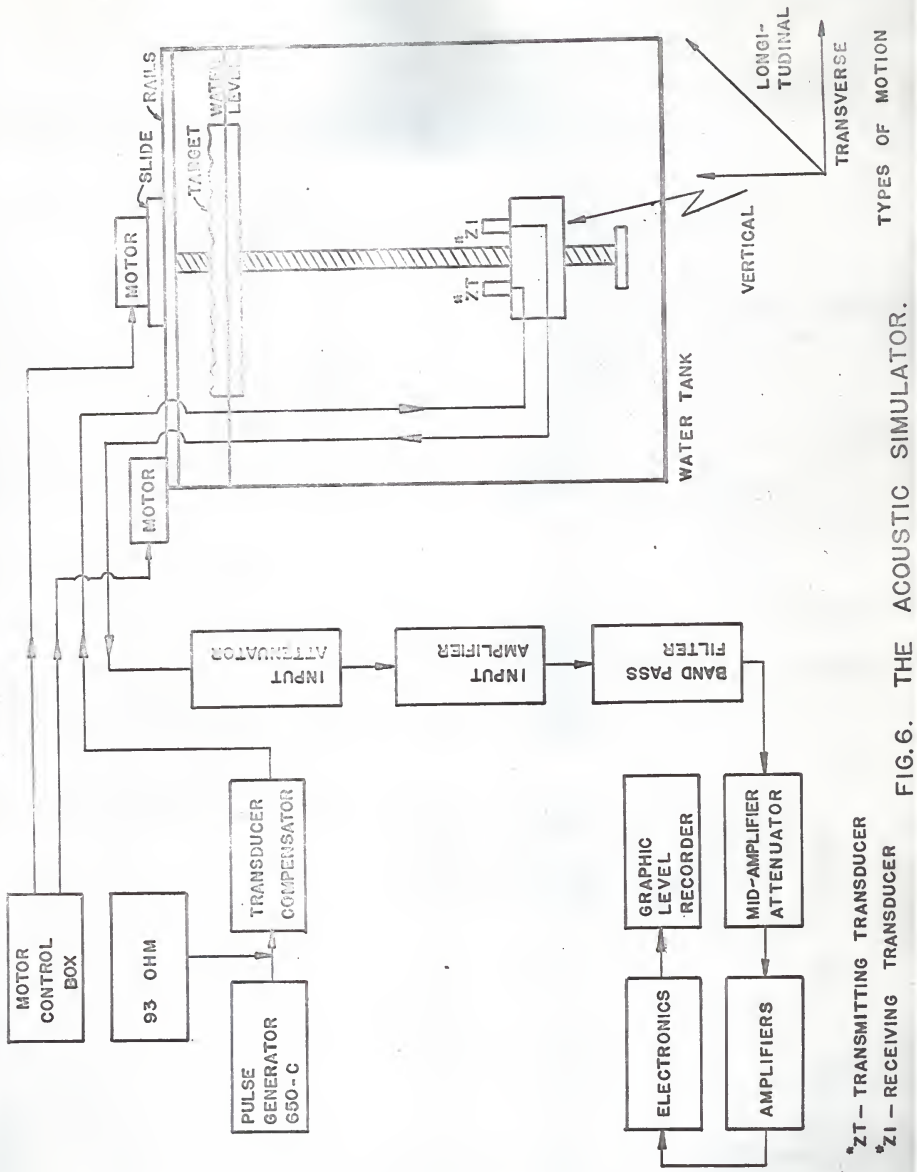


A block diagram of the equipment which was used for the acoustic simulation of a rough layer is shown in Fig. 6. The acoustic waves are generated in a large water filled tank by means of an electro-acoustic transducer, which is driven by an electronic oscillator. The transducer is capable of being moved in three orthogonal directions relative to the target; it can also be rotated about horizontal and vertical axes to give any desired angle of incidence. A brief description of the experimental equipment is given below. Details concerning the calibration and use of the apparatus can be found in the KSU Technical Report EE-TR-1 [39].

\subsection{Acoustic Simulator Components.}

(A) Pulsed Oscillator: The high powered pulse oscillator (PG 650-c, Model 2, Arenberg Ultrasonic Lab., Jamaica Plain, Massachusetts) is a variable frequency, pulse modulated radio frequency oscillator capable of delivering 300 volts peak to peak into a $93 \mathrm{ohm}$ load resistor. The oscillator can deliver output pulses that vary in width from $2 \mu \mathrm{sec}$. to $100 \mu \mathrm{sec}$. over a frequency range of $0.5 \mathrm{Mc} / \mathrm{sec}$. to $5.0 \mathrm{Mc} / \mathrm{sec}$.

(B) Transducers: All acoustic measurements were made by means of piezoelectric barium titnate transducers (piston shaped), manufactured by Branson Instruments Manufacturing , Stanford, Connecticut. Four pairs of transducers rated at the following frequencies were used: $1.0 \mathrm{mc}, 1.6 \mathrm{Mc}, 2.25 \mathrm{Mc}$, and $3.5 \mathrm{Mc}$. Each pair of transducers consists of a transmitter and a receiver 
designated by $\mathrm{ZT}$ and $\mathrm{ZI}$, respectively. Table I gives the diameter and beamwidth of the respective transducers.

Table I. Beamwidth of transducers.

\begin{tabular}{|c|c|c|c|}
\hline Table I. & Beamwidth of transducers. \\
\hline Frequency in Mc. & $\begin{array}{l}\text { Diameter of active } \\
\text { element in inches. }\end{array}$ & $\begin{array}{l}\text { Holder diameter } \\
\text { in inches. }\end{array}$ & $\begin{array}{l}\text { Beamwidth } \\
\text { in degrees. }\end{array}$ \\
\hline 1.00 & $7 / 8$ & $5 / 4$ & 3.1 \\
\hline 1.60 & $1 / 2$ & $3 / 4$ & 3.8 \\
\hline 2.25 & $1 / 2$ & $3 / 4$ & 2.8 \\
\hline 3.50 & $1 / 2$ & $3 / 4$ & 1.9 \\
\hline
\end{tabular}

(C) Transducer Compensator: Functionally, the transducers operate best at mechanical and electrical resonance. Electrically the transducer appears to be a capacitor shunted by a small conductance. Electrical resonance is, therefore, achieved by adding the proper inductance in parallel to cancel the total capacitive reactance. Without such compensation, the large capacitance of the transducer cable will "pull" the oscillator frequency out of the range of frequencies marked on the oscillator coils. A transmitting transducer compensator obtains this resonance, so that the ultrasonic oscillator can see at any frequency a relatively non-reactive load.

(D) Receivers and Detectors: In Fig. 6 it is shown that the reflected acoustic waves are intercepted by the receiving transducer (which is positioned next to the transmitting transducer) and converted there into an electrical pulse which is then transferred through an input attenuator, an input amplifier, a band 
pass filter, a mid-amplifier attenuator, and two wide-band amplifiers to the block marked "electronics". The "electronics" is composed of the following:

(1) Recycle gate generator.

(2) Amplifier gate generator.

(3) Gated amplifier.

(4) Detector.

(5) Video amplifier.

(6) Boxcar circuit.

(E) Recording Device: The acoustic return data was recorded by the use of General Radio Type 1521-A graphic level recorder. This recorder has an input resistance of 1000 ohms and is driven by $a \mathrm{~d}-\mathrm{c}$ analog voltage from the boxcar detector. The voltage normally ranges from 0 to 0.8 volts, providing a maximum stylus deflection of four inches on the chart.

(F) Motor Control: All control of the scanning mechanism is normally done from a remote motor control box.

(G) The tank is 6 feet long, 5 feet wide, and 5 feet deep. It is constructed of $1 / 8$ inch galvanized steel sheets.

(H) Scanning Mechanism: The tank scanning mechanism is built on a steel slide which is supported by wheels mounted on two parallel angle iron rails at the top of the tank. Thus the tank scanning mechanism can be placed at any desired distance from the target up to a maximum of 44 inches. 
The scanning carriage is mounted upon the steel slide so that east-west motion is given to the scanning carriage by an a-c split phase motor which is fixed to one end of the slide. Vertical motion of the transducers is obtained through another a-c split phase motor supported by the carriage and driving a vertical screw shaft. On this shaft ride the transducer mount and the transducer vertical scan assumbly.

\subsection{Target Description.}

The layer target was made from a plexiglas sheet 48 inches long, 6 inches wide, and 2 inches thick. One face of the plexiglas sheet was machined to give a one dimensionally rough surface, leaving the other surface smooth, as shown in Fig. 7. The purpose of having the roughness in only one dimension ( $z$ coordinate) rather than the more realistic two dimensional roughness (in $\mathrm{y}$ and $\mathrm{z}$ coordinates), is to have a better control over the statistics of the roughness.

The heights of the rough surface were chosen from a normal distribution having a standard deviation $\sigma_{z}=0.05$ inches. These heights were then arranged (by trial and error) to give a random curve having a Gaussian autocorrelation function with a correlation distance $T=0.15$ inches. This particular correlation function was obtained by arranging the heights, such that there were not many sudden variations in the slopes of the curve thus generated. The random curve was then traced on the edge of the plexiglas, which was cut by a shaping machine. Finally, the machined rough surface was sampled by a profilometer (apparatus 


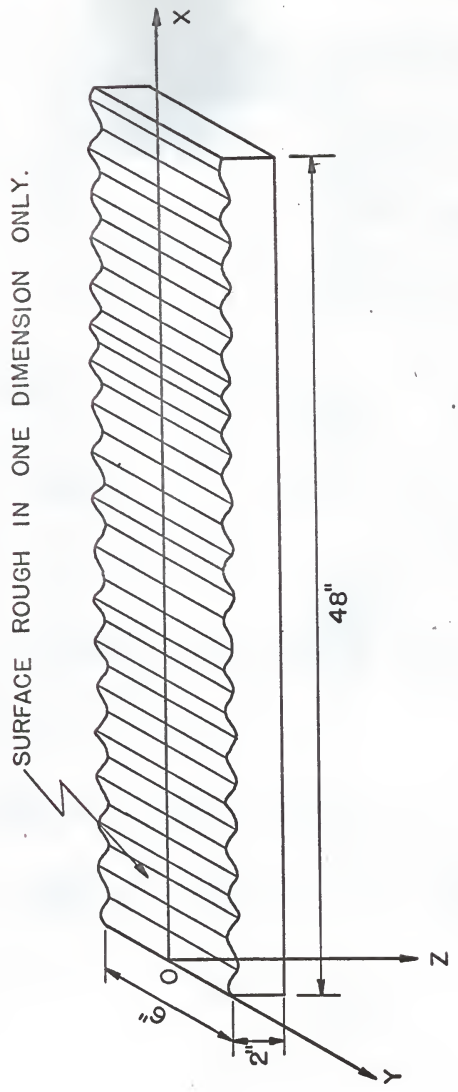

$\frac{1}{2}$
$\frac{1}{4}$
$\frac{1}{4}$
$\frac{1}{4}$
$\frac{1}{4}$
$\frac{1}{4}$ 


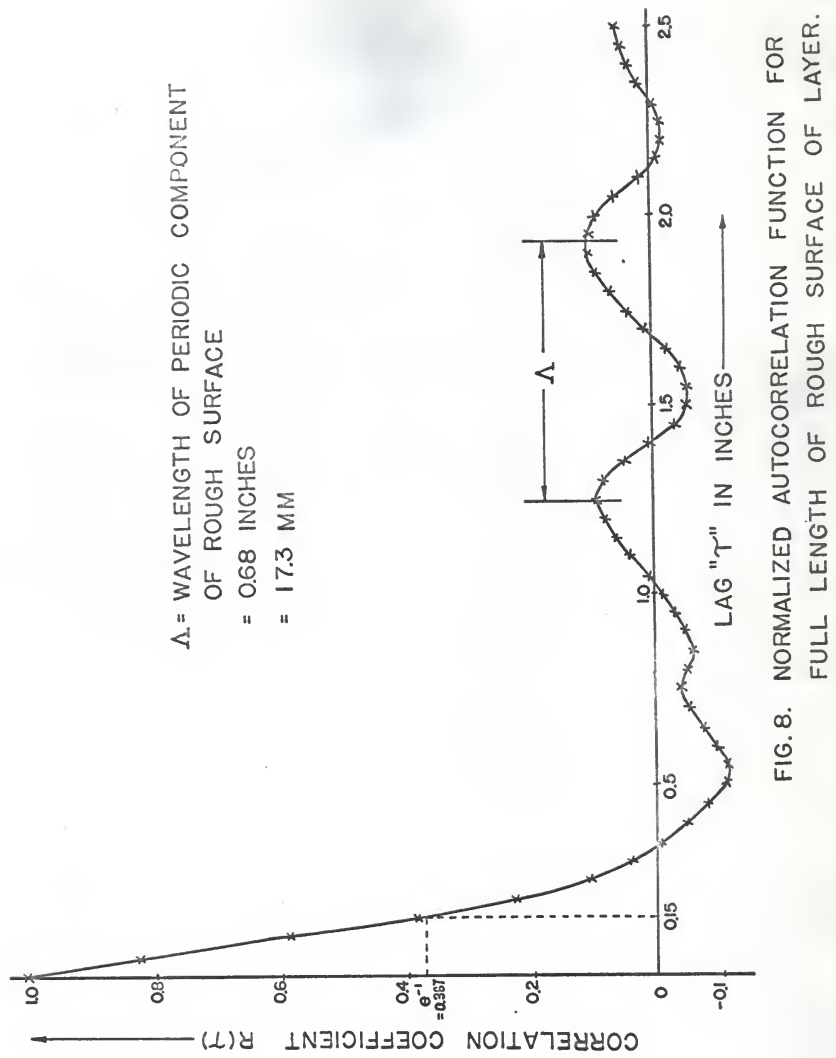


for determining the profile of a rough surface) and the statistics redetermined from the measured heights. The measured standard deviation $\sigma_{z}$ and correlation distance $T$ were 0.046 inches, and 0.150 inches respectively. The measured autocorrelation function $R(\tau)$ (Normalized) is shown in Fig. 8.

\subsection{Procedure for the Experiment.}

The target was mounted on supports and placed flat on the surface of the water in the tank, such that one surface (front) was within the water and the other surface (back) was exposed to air, as shown in Fig. 6. The purpose of this particular configuration was to simulate the perfectly conducting medium behind the rough surface, since the reflection coefficient at the plexiglas-air interface approaches unity.

The backscattered signal was recorded for two different configurations of the layer. In one configuration, which shall be termed the "rough back", the front surface of the layer was smooth and the back surface rough. In the other configuration, which shall be termed "rough front", the front surface of the layer was rough and the back surface smooth. In each case the backscattered signal from the rough interface only was recorded, the signal from the smooth interface being gated out.

Transducer Positioning: The transducers were so positioned that they were focussed on the layer when placed at a distance of 32 inches from it. The transducer assembly was moved from one end 
of layer to the other, so that the full length of layer was scanned. The backscattered signal was recorded by the graphic level recorder during the scan.

Frequency and Angle of Incidence: In the "rough back" configuration of the layer, the frequencies used were: $0.72 \mathrm{Mc}, 1.0 \mathrm{Mc}$, $1.28 \mathrm{Mc}, 1.6 \mathrm{Mc}, 1.9 \mathrm{Mc}, 2.25 \mathrm{Mc}, 3.0 \mathrm{Mc}$ and $3.5 \mathrm{Mc}$. For the "rough front" configuration the frequencies used were: $1.0 \mathrm{Mc}$, $1.6 \mathrm{Mc}, 2.25 \mathrm{Mc}$ and $3.5 \mathrm{Mc}$.

Four different angles of incidence, $0,5,10$, and 20 (at front surface of layer) were used for each configuration of the layer. The distance of the transducers from the front interface of the layer was always kept constant at 32 inches, as stated earlier.

Pulse Length: The pulse length chosen was $20 \mu \mathrm{sec} .$, which is less than the time taken (about $30 \mu \mathrm{sec}$ ) for the pulse front to travel from the front surface of the layer to the back surface, and back again to the front surface. This way two separate and distinct signals from the two interfaces of the layer were obtained at the receiver. These two backscattered signals were separated in time by about $10 \mathrm{\mu sec}$ for normal incidence.

The pulse length was deliberately chosen to be less than $30 \mu \mathrm{sec}$, so that the backscattered signal from the rough interface of the layer could be recorded separate from the backscattered signal by the smooth interface.

Recording and Measurements: The value of the backscattered signal at 32 inches from a water-air interface was recorded for each 
frequency. These values were then used to normalize the backscattered signal from the layer. The value of the recorded signal directly gives $\sqrt{\sigma_{0}}$, the square root of the normalized radar cross section of the layer. Therefore, the square of the recorded signal gives $\sigma_{0}$. The mean value and variance of $\sigma_{0}$ were calculated for each set of recorded data. Further details and sample calculations for $\sigma_{0}$ are given in Appendix II.

Limitations for $20^{\circ}$ : For the $20^{\circ}$ angle of incidence, the backscattered signal from only four-fifths length of the layer could be recorded due to the limitations in the transverse motion of the scanning mechanism. The measured autocorrelation function $R(\tau)$ (normalized) for this part of the rough surface of layer is shown in Fig. 9: it is very similar to the measured autocorrelation for the full layer shown in Fig. 8. The mean value and variance of the heights of the rough surface are almost the same for the two cases.

It may be pointed out that the sound wave transmitted into the plexiglas layer at an angle of incidence other than normal has two modes of propagation: the longitudinal mode and the transverse (shear) mode. At large angles of incidence a considerable amount of sound energy in plexiglas will be propagated in the transverse mode. Therefore, the measured backscattered data for the "rough back" configuration of the layer at $20^{\circ}$ angle of incidence may not be very accurate.

\subsection{Results:}

The results of the "rough back" configuration of the layer 


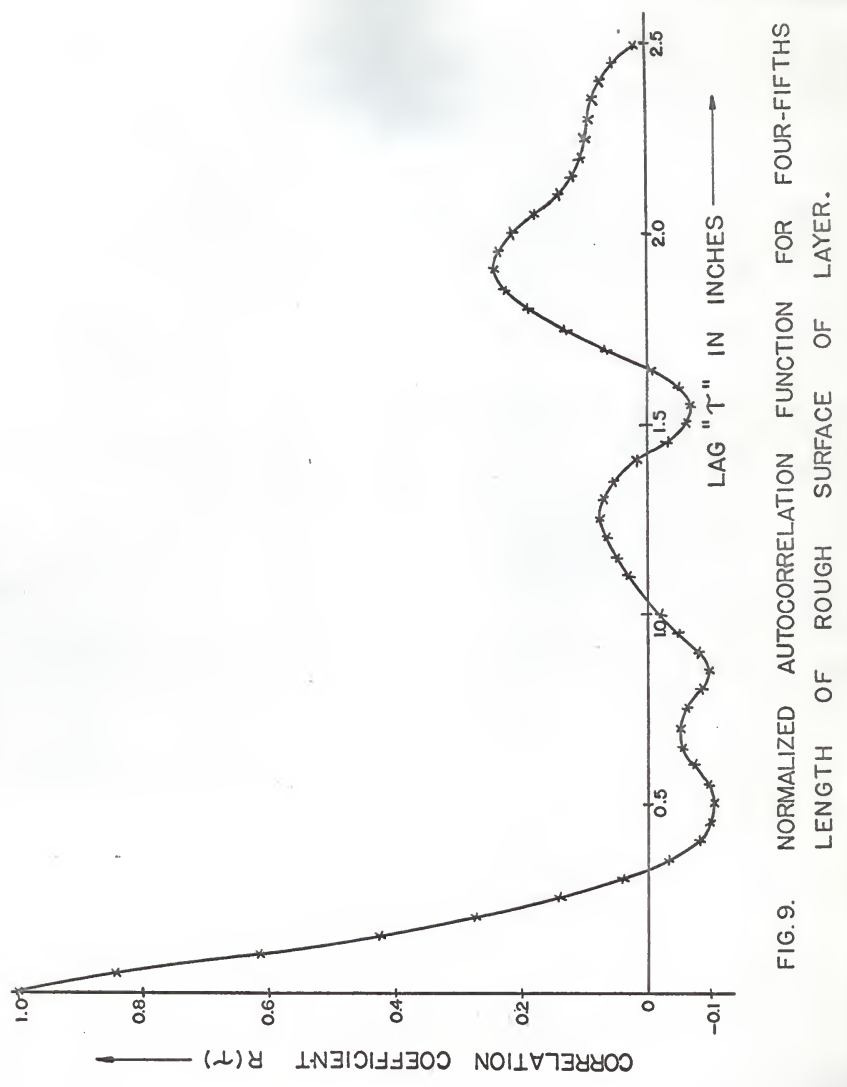




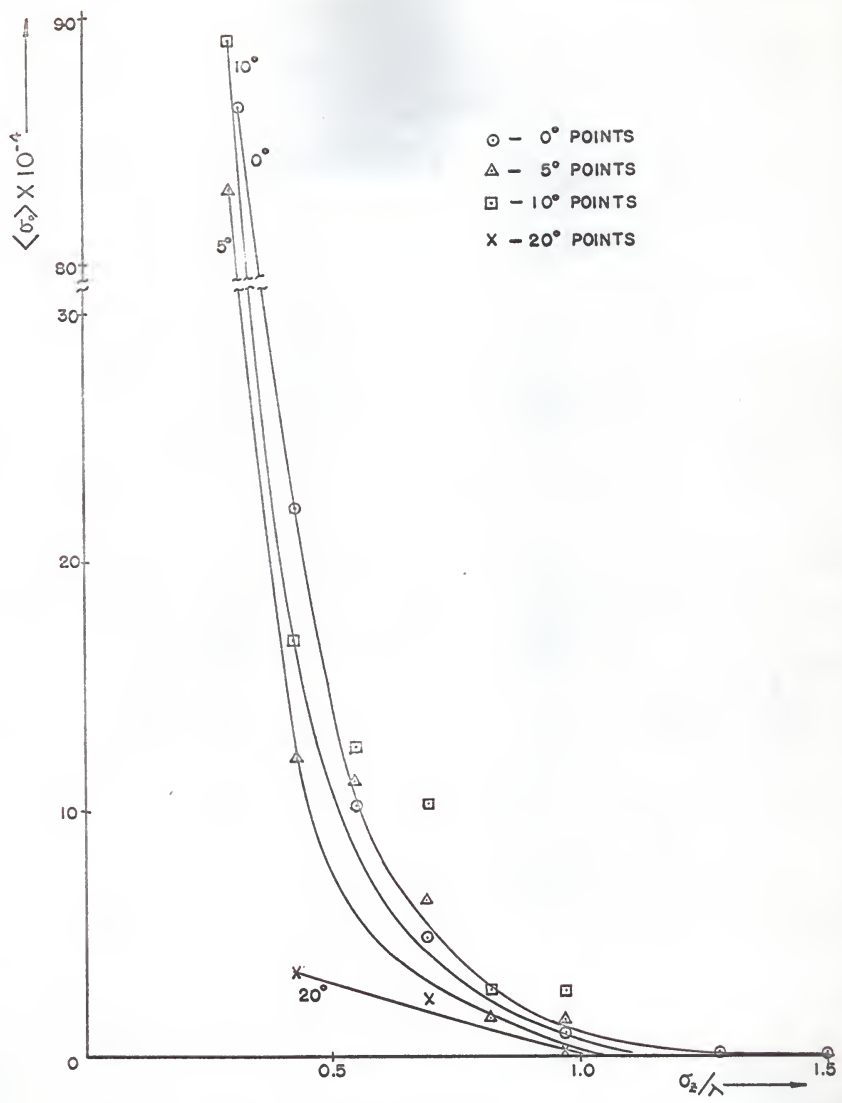

FIG.IO. RADAR CROSS SECTION VERSUS $\sigma_{z} / \lambda$ FOR ROUGH BACK CONFIGURATION OF LAYER. 


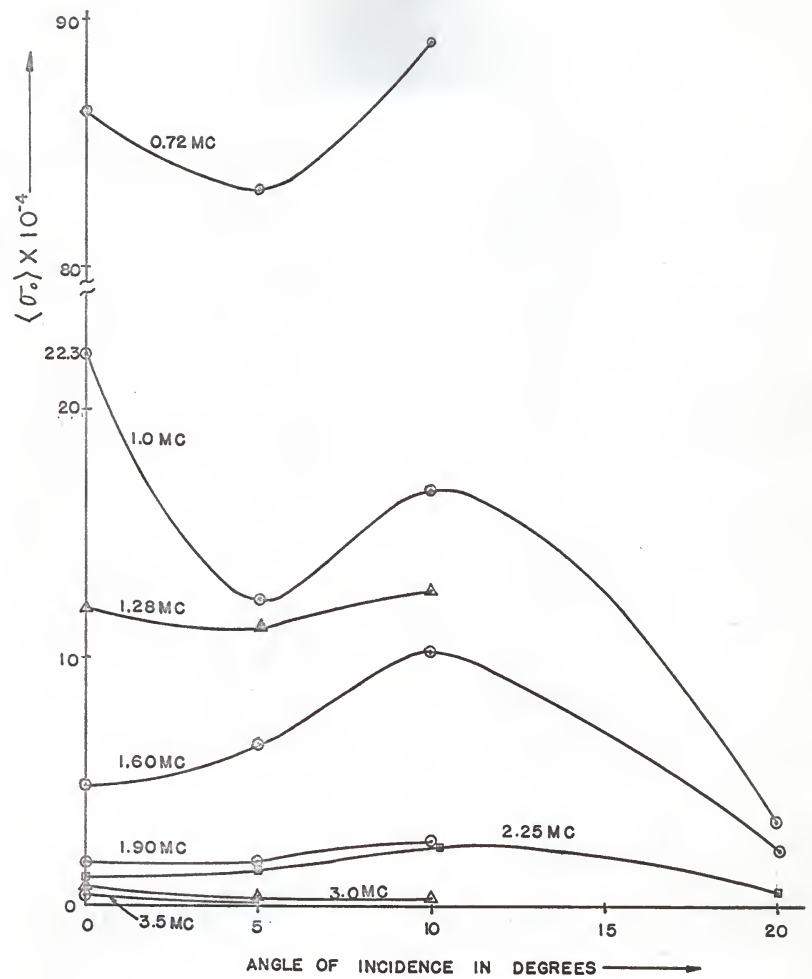

FIG.II. RADAR CROSS SECTION VERSUS ANGLE OF INCIDENCE FOR ROUGH BACK CONFIGURATION OF LAYER. 


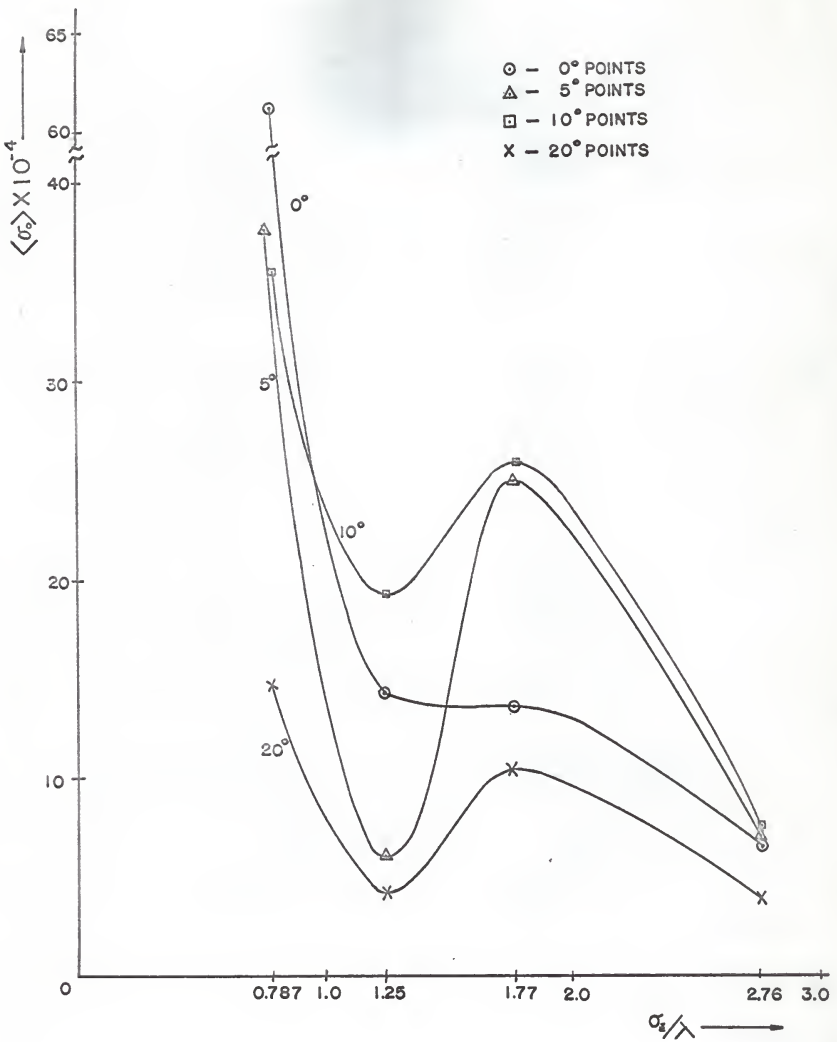

FIG.12. RADAR CROSS SECTION VERSUS $\sigma_{i} / \lambda$ FOR ROUGH FRONT CONFIGURATION OF LAYER. 


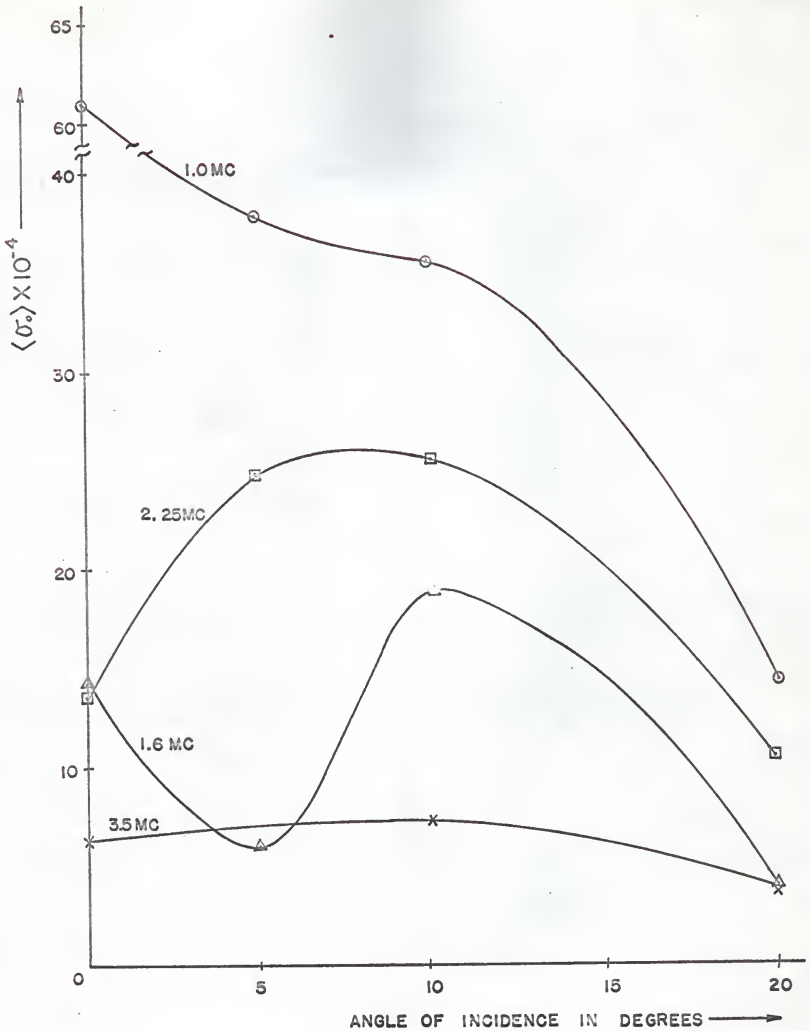

FIG.13. RADAR CROSS SECTION VERSUS ANGLE OF INCIDENCE FOR ROUGH FRONT CONFIGURATION CF LAYER. 
have been summarized in Figs. 10 and 11 . of these, Fig. 10 gives the mean value of $\sigma_{0}$ (normalized radar cross section) as a function of $\sigma_{z} / \lambda$ showing four curves for angles of incidence of $0^{\circ}$, $5^{\circ}, 10^{\circ}$ and $20^{\circ}$, respectively. Here $\sigma_{z} / \lambda$ is the ratio of $\sigma_{z}$, the standard deviation of the heights of rough surface of layer, to $\lambda$, the wavelength of the incident wave in plexiglas. The various values of $\sigma_{z} / \lambda$ are given in Table III, Appendix I. Fig. 11 shows the dependence of $\left\langle\sigma_{0}\right\rangle$ on angle of incidence, with frequency as a parameter. The measured mean values and standard deviation of $\sigma_{0}$ are tabulated in Table IV, Appendix III.

Similarly, Figs. 12 and 13, summarize the results for the "rough front" configuration of the layer. In this case $\sigma_{z} / \lambda$ is the ratio of the standard deviation of the heights of rough surface of layer to the wavelength of the incident wave in water. The values of $\sigma_{z} / \lambda$ used are given in Table II, Appendix I. Again the measured mean values and standard deviation of $\sigma_{0}$ are tabulated in Table V, Appendix III.

\subsection{Discussion of Results.}

The dependence of $\left\langle\sigma_{0}\right\rangle$ on $\sigma_{z} / \lambda$ and the angle of incidence as shown in Figs. 10 to 13 seems to be in accordance with the theoretical results of Chapter 3 , as seen below.

\section{(A) Rough Back Configuration of Layer.}

(1) Dependence on $\sigma_{z} / \lambda$ : Figure 10 shows that for a particular angle of incidence, as $\sigma_{z} / \lambda$ increases (or in other words as the surface becomes more rough), the value of $\left\langle\sigma_{0}\right\rangle$ decreases. This 
should be expected from the theoretical results (Eq. 64, Chapter 3) as discussed in Chapter 3. It is to be noted that in Fig. 10 the value of $\left\langle\sigma_{0}\right\rangle$ is maximum for $\theta=0^{\circ}$, falls for $\theta=5^{\circ}$, but rises for $\theta=10^{\circ}$, and finally falls again for $\theta=20^{\circ}$. In short, almost all the curves in Fig. 11 are peaked at $\theta=10^{\circ}$. The probable reason for this behaviour is the fact that the rough surface has more facets with $10^{\circ}$ slope than facets with $5^{\circ}$ slope. A check of the number of facets having $5^{\circ}$ and $10^{\circ}$ slopes, respectively, confirmed this fact. The slopes were calculated by sampling the rough surface at intervals of 0.05 inches, finding the difference in heights of the adjacent sampled points and then dividing this difference by 0.05 inches (the sampling distance). From these calculated slopes, the total number of $5^{\circ}$ and $10^{\circ}$ slopes, respectively, were counted and finally compared.

The theoretical results of Chapter 3 indicate that as the angle of incidence $\theta$ increases, the value of $\left\langle\sigma_{0}\right\rangle$ should decrease. However, these theoretical results were obtained for a surface having a normal distribution of slopes, while the rough surface of the layer used for the experimental work does not have a normal distribution of the slopes, as is evident from the fact that the $10^{\circ}$ slopes are larger in number than the $5^{\circ}$ slopes. Therefore, there is reason to expect some differences between the experimental results and the theory.

\section{(B) Rough Front Configuration of Layer.}

(1) Dependence on $\sigma z / \lambda$ : In Fig. 12, it is noted that in agreement with the theory, the value of $\left\langle\sigma_{0}\right\rangle$ decreases with increase of $\sigma_{z} / \lambda$ (i.e., as the surface becomes more rough) as $\sigma_{z} / \lambda$ changes 
from 0.787 to 1.250 ; however, for $\sigma_{z} / \lambda=1.770$ the value of $\left\langle\sigma_{0}\right.$ > increases, although at $\sigma_{z} / \lambda=2.760,\left\langle\sigma_{0}\right\rangle$ decreases again.

This peaking of the curves at $\sigma_{z} / \lambda=1.770$ is probably due to some periodicity in the rough surface of layer. As shown in Appendix IV, the equation from grating theory indicates that if $\lambda / \Lambda$, the ratio of the wavelength of the incident radiation to the wavelength of periodicity of the surface, is small, then the incident wave will be broken up into many scattered waves or modes. The autocorrelation function of the rough surface of layer, given in Fig. 8, does indicate that a periodic surface has been superimposed on the randomly rough surface. Figure 8 shows that the wavelength $\Lambda$ of the periodic surface is of the order of $17.3 \mathrm{~mm}$. At $2.25 \mathrm{MC}\left(\sigma_{z} / \lambda=1.770\right)$, the wavelength of sound in water is $\lambda=0.666 \mathrm{~mm}$. The ratio $\frac{\lambda}{\Lambda}=\frac{0.666}{17.3}=\frac{1}{2} 6 \ll 1$, thus satisfying the condition stated above. The scattering pattern should therefore consist of the superposition of the multi-lobed modes of the periodic component of the rough surface and the diffuse scattering pattern of the random component of the rough surface. The resultant scattering pattern will therefore have a number of directional maximas. It is possible that the measured backscattered data at $\sigma / \lambda=1.770$ was on one of these maximas, whereas the measured backscattered data for the adjacent value of $\sigma_{z} / \lambda=$ 1.25 was not on one of these maximas.

Therefore, one probable reason for the peaking of the curves at $\sigma_{z} / \lambda=1.770$ (frequency $=2.25 \mathrm{Mc}$ ) is the grating effect of the periodic component of the rough surface. 
(2) Dependence on Angle of Incidence $\theta$ : Figure 13 shows that the values of $\left\langle\sigma_{0}\right\rangle$ at $\theta=5^{\circ}$ and $\theta=10^{\circ}$ are comparable for all cases except for the case of $1.6 \mathrm{Mc}$. This should be expected, because the rough surface has a larger number of facets having $10^{\circ}$ slope compared to facets having $5^{\circ}$ slope, as mentioned earlier. In case of the $1.6 \mathrm{Mc}$ curve, there is a big increase in $\left\langle\sigma_{0}\right\rangle$ as $\theta$ is increased from $5^{\circ}$ to $10^{\circ}$; this difference in the behaviour is probably due to the grating effect of the periodic component of the rough surface as already explained. Otherwise, all the four curves do have a tendency of decreasing with the increase in $\theta$.

Theory indicates that the value of $\left\langle\sigma_{0}\right\rangle$ should fall steadily with the increase in $\theta$, for all frequencies. In case of the curves of Fig. 13, this holds except for $2.25 \mathrm{Mc}$, the difference being due to the reasons given above. 


\section{CONCLUSIONS}

The field scattered from a rough surface is known to be a complicated function of the parameters involved, e.g. angles of incidence and scatter, polarization, frequency, dielectric and statistical properties of the scatterer, etc. The problem is more complicated, however, when the target consists of a rough layer covering a thick core, this being the simplest form of the general multi-layer problem. To better understand the scattering behaviour of a rough layer, both theoretical as well as experimental work was attempted.

In Chapter 3 expressions were derived for the mean radar cross section (normalized) $\left\langle\sigma_{0}\right\rangle$ of a rough layer. The incident wave was considered to be a pulse of the beamwidth limited type, so as to conform with actual practice. To simplify the analysis the rough layer was assumed to have a smooth frontsurface and a rough back surface. Two important restrictions were:

(1) The beamwidth of the incident wave is much smaller than the mean depth of the layer.

(2) The mean depth of layer is much greater than the wavelength of the incident wave within the layer; in other words, within the layer, the backscattered electric field at the smooth front interface is in the far zone of diffration.

From the theoretical expressions obtained for $\left\langle\sigma_{0}\right\rangle$ in Chapter 3 , it is reasonable to conclude that the value of $\left\langle\sigma_{0}\right\rangle$ decreases as:

(1) the angle of incidence departs from normal,

(2) the roughness of the rough surface of layer increases. 
The limitations of the particular model of rough layer chosen for the theoretical analysis have been noted, with a view to help improve future work. This indeed is a first step towards the solution of a more general rough layer problem.

The experimental work was done by acoustic simulation technique. A rough layer was made from 2 inches thick plexiglas sheet, one surface of which was machined to give a layer with one surface rough and the other surface smooth. Backscatter measurements were made for two configurations of the layer:

(1) the back surface rough and the front surface smooth. (2) the front surface rough and the back surface smooth. The dependence of mean radar cross section of layer $\left\langle\sigma_{0}\right\rangle$ (for both configurations) on the angle of incidence $\theta$ and $\sigma_{z} / \lambda$, the ratio of the standard deviation of the heights of the rough surface to the wavelength of the wave incident on the rough surface, is plotted in Figs. 10 to 13, Chapter 4. From these curves it may be concluded that $\left\langle\sigma_{0}\right\rangle$ decreases with:

(1) increase in roughness of layer (i.e., increase of $\sigma_{z} / \lambda$ ),

(2) increase in angle of incidence.

This is in agreement with the theoretical results of chapter 3.

Various limitations of the measured experimental data for the rough plexiglas layer by acoustic simulation were:

(1) Plexiglas is a lossy substance.

(2) At angles of incidence other than normal, the sound energy transmitted into the plexiglas layer propagates in both the longitudinal and transverse modes, rather than the desired longitudinal mode only. These 
limitations are bound to affect the measured results while they have been neglected in the theoretical study.

(3) The rough surface of the layer, which was obtained by machining it on a shaping machine, was obviously not random enough, since it was found to have a periodic component superimposed on a randomly rough component; moreover, the slopes of the rough surface were not distributed normally but had a "peak" at $10^{\circ}$ as well as $0^{\circ}$. Only a partial attempt was made to compare the experimental results with the theoretical results and no direct comparison could be made due to the limitations of the measured data, as described above. The experiment also violated the assumption of the theory that within the layer, the backscattered electric field at the smooth front interface is in the far zone of diffraction, i.e., the mean depth of layer $d \gg \lambda$ and $d \gg$ the incident beamwidth. The violation of this assumption was unavoidable because :

(1) d could not be increased beyond 2 inches; plexiglas sheets thicker than 2 inches are not easily available commercially. Even if they were available, the wave propagating within it would be attenuated considerably, thus violating the assumption that the layer is lossless.

(2) $\lambda$ and beamwidth could not be decreased further; the frequency range was limited due to the limited number of transducers and the pulsed oscillator available. 


\section{RECOMMENDATIONS FOR FUTURE STUDIES}

In Chapter 3 the normalized radar cross section of a rough layer with the front surface smooth and the back surface rough has been derived with many simplifying restrictions; however, it seems to be the first such attempt to solve the rough layer problem. The literature was thoroughly reviewed; it seems that no previous attempt has been made to solve the problem of backscatter of electromagnetic waves from a rough layer.

The model to be considered next should consist of a rough layer having the front surface rough and the back surface smooth, assuming as before that the depth of layer is much greater than the incident wavelength within the layer so that the back surface of the layer may be considered to lie in the far zone of diffraction. It may be pointed out that the theoretical analysis of this model will be much more complex compared to the model chosen in Chapter 3. A statistical approach to this problem is worth investigating; it might prove to be less complex and yield more useful results as compared to the physical optics method. Parks [30] gives a statistical solution for backscatter from sea, in the near zone of diffraction. This approach may be extended to the case of backscatter from a rough layer. The case of a single rough layer may be said to be completely solved only when the solution is found for a layer having both the boundaries randomly rough and no restriction on the mean depth.

Meanwhile an experimental approach may be envisaged. Instead of acoustic simulation of the electromagnetic problem, it is 
recommended that experiments should be conducted using microwaves. This will give a greater flexibility in choosing the material for the layer, and will also yield more quantitative results. While choosing the layer material for acoustic simulation it was observed that most of the available materials (solids) were lossy and had a very high reflection coefficient when the sound wave was incident from water. Moreover, the energy transmitted by the longitudinal acoustic wave into a solid from a liquid-solid interface has two modes of propagation, the longitudinal mode and the transverse (shear) mode. This causes an error in the observed results. At large angles of incidence most of the sound energy transmitted into the solid propagates in the transverse mode, rather than the longitudinal mode. Therefore, care should be taken not to use large angles of incidence, the limit depending upon the actual liquid and solid used.

Various models of layer should be made, their backscattered signal recorded, and their average radar cross section calculated. The materials as well as the statistics of the target may be varied. A complete set of such experiments will yield considerable information on the statistics and electrical properties of the layer material. 
APPENDIX I

Target Construction, Properties \& Statistics

\section{Target Construction.}

The heights of the rough surface (one dimensional) of the layer were chosen to belong to a normal distribution with standard deviation $\sigma_{z}=0.05^{\prime \prime}$. Only the area under the normal curve lying between $-3 \sigma_{z}$ and $+3 \sigma_{z}$ was considered. These heights were then arranged to give a Gaussian covariance function (normalized autocorrelation function) with a correlation distance of 0.15 inch. This particular correlation function was obtained by trial and error using a 1620 IBM computer. It was noted that the heights had to be arranged in such a manner, that there were not many sudden variations in the slopes of the surface thus generated. This random curve of known statistics was then traced on the edge of the plexiglas, which was then cut by a shaping machine; care being taken to follow the curve as closely as possible.

After the plexiglas had been cut, the rough surface was sampled and its mean value, standard deviation and normalized autocorrelation function was redetermined. The profilometer (apparatus for determining the profile of a rough surface) used for sampling the target was accurate up to one thousandth of an inch. The sampling was done at intervals of 0.05 inch. The measured standard deviation and correlation distance are 0.0465 . inches $(1.18 \mathrm{~mm})$ and 0.150 inches $(3.81 \mathrm{~mm})$, respectively. The 
normalized autocorrelation function is given in Fig. 8, Chapter 4. The mean value was found to lie almost midway between the highest and lowest points sampled.

The estimate of the normalized correlation function was calculated using the following expression:

$$
R(\tau \Delta x)=\frac{\frac{1}{\bar{N}-\tau} \sum_{i=1}^{N-\tau}\left(h_{i}-\bar{m}\right)\left(h_{i}+\tau-\bar{m}\right)}{\frac{1}{\bar{N}} \sum_{i=1}^{N}\left(h_{i}-\bar{m}\right)^{2}}
$$

where

$$
\begin{aligned}
& \mathrm{h}_{i} \text { is the } i^{\text {th }} \text { height in the series of } \mathrm{N} \text { points. } \\
& \tau \quad \text { is the lag. } \\
& \Delta \mathrm{x} \text { is the sampling interval. } \\
& \overline{\mathrm{m}}=\frac{1}{\mathrm{~N}} \sum_{i=1}^{N} \mathrm{~h}_{i} \text { is the sample mean. }
\end{aligned}
$$

At normal incidence, the diameter of the area illuminated on the target varies from 1 to 2 inches, depending upon the particular set of transducers used, the distance between the transducers and the target being 32 inches. Taking the mean value of the diameter of illuminated area to be 1.5 inches, the correlation distance of 0.15 inches was chosen to be about one tenth of this mean beam width. The value of $\sigma_{z}=0.15$ inches was chosen to give a very rough surface for all the four frequencies 
used; the criterion for a very rough surface being $\frac{\sigma_{z}}{\lambda}>0.16$. Tables II and III list the values of $\lambda$ and $\frac{\sigma_{z}}{\lambda}$ for water and plexiglas, respectively.

Table II. Wavelength of Sound in Water.

\begin{tabular}{|c|c|c|}
\hline Frequency in Mc. & $\lambda$ in $\mathrm{mm}$ & Ratio $\sigma_{\mathrm{z}} / \lambda\left(\sigma_{\mathrm{z}}=1.18 \mathrm{~mm}\right)$ \\
\hline 1.00 & 1.500 & 0.787 \\
\hline 1.60 & 0.946 & 1.250 \\
\hline 2.25 & 0.666 & 1.770 \\
\hline 3.50 & 0.428 & 2.760 \\
\hline
\end{tabular}

The velocity of sound in water was taken to be 1500 meters/sec.

Table III. Wavelength of Sound in Plexiglas.

\begin{tabular}{|c|c|c|}
\hline Frequency in Mc. & $\lambda$ in $\mathrm{mm}$ & Ratio $\sigma_{\mathrm{z}} / \lambda\left(\sigma_{\mathrm{z}}=1.18 \mathrm{~mm}\right)$ \\
\hline 0.72 & 3.860 & 0.306 \\
\hline 1.00 & 2.780 & 0.425 \\
\hline 1.28 & 2.170 & 0.544 \\
\hline 1.60 & 1.740 & 0.679 \\
\hline 1.90 & 1.465 & 0.806 \\
\hline 2.25 & 1.235 & 0.955 \\
\hline 3.00 & 0.927 & 1.270 \\
\hline 3.50 & 0.794 & 1.490 \\
\hline
\end{tabular}

The longitudinal velocity of sound in plexiglas was experimentally determined to be 2780 meters/sec. 
Reflection Coefficient at Water-Plexiglas Interface: At normal incidence, the acoustic (characteristic) impedance $\mathrm{z}$ of a material is given by

$$
z=\rho C
$$

where

$$
\begin{aligned}
\rho= & \text { density of material. } \\
\mathrm{C}= & \text { longitudinal velocity of sound in the } \\
& \text { material. }
\end{aligned}
$$

For water, the acoustic impedance is

$$
\mathrm{z}_{\mathrm{W}}=\rho_{1} \mathrm{C}_{\mathrm{W}}=15 \times 10^{4}
$$

where

$$
\begin{aligned}
& \rho_{1}=1.0 \mathrm{gms} / \mathrm{cm}^{3} \\
& C_{w}=15 \times 10^{4} \mathrm{~cm} / \mathrm{sec} .
\end{aligned}
$$

For plexiglas, the acoustic impedance is

$$
\mathrm{z}_{\mathrm{p}}=\rho_{2} \mathrm{C}_{\mathrm{p}}=33.40 \times 10^{4}
$$

where

$$
\begin{aligned}
& \rho_{2}=1.2 \mathrm{gms} / \mathrm{cm}^{3} \\
& c_{p}=27.8 \times 10^{4} \mathrm{~cm} / \mathrm{sec} .
\end{aligned}
$$

The reflection coefficient $\Gamma$ for a plane sound wave incident from water on a water-plexiglas interface, is given by

$$
\begin{aligned}
\Gamma & =\frac{z_{p}-z_{W}}{z_{p}+z_{W}} \\
& =0.380 .
\end{aligned}
$$

It may be mentioned that many man hours were spent in generating a random curve of known statistics, constructing this 
curve on plexiglas, and finally re-calculating the statistics of the rough surface thus obtained. Two computer programs used for computing the mean value, variance, normalized autocorrelation function and the radar cross section, respectively, of the one dimensionally rough surface, are given below.

COMPUTER PROGRAM FOR DETERMINATION OF TARGET STATISTICS.

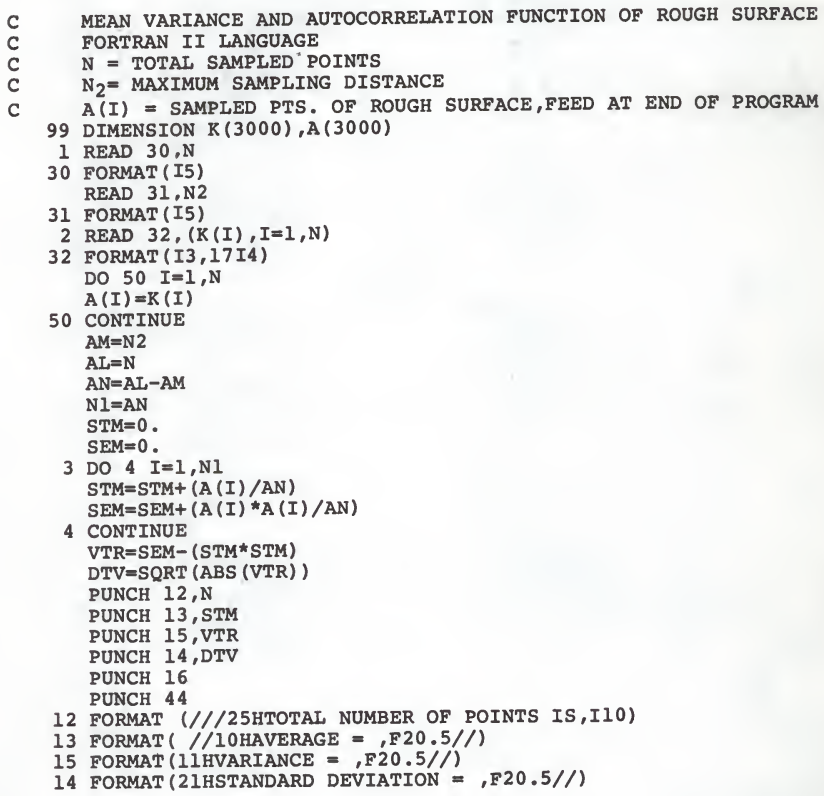


16 FORMAT (///36HNORMALLIZED AUTO CORRELATION FUNCTION)

44 FORMAT ( //16X, 1HT , 31X, $4 \mathrm{HZ}(\mathrm{T}) /$ )

$\mathrm{L}=0$

25 RES $=0$.

DO $8 \quad I=1, N 1$

$\mathrm{J}=\mathrm{L}+\mathrm{I}$

RES $=$ RES $+(A(I) * A(J)) / A N$

8 CONTINUE

$A U T O=(R E S-(S T M * S T M)) /(D T V * D T V)$

$\mathrm{R}=\mathrm{L}$

PUNCH 20,R,AUTO

20 FORMAT $(4 \mathrm{X}, \mathrm{F} 15.2,6 \mathrm{X}, \mathrm{F} 30.10)$

$\mathrm{L}=\mathrm{L}+1$

IF (N2-L) $27,25,25$

27 STOP

END

COMPUTER PROGRAM FOR CALCULATION OF NORMALIZED RADAR CROSS SECTION OF TARGET.

C
C
C
C
C
C
C
C

FIND REFLECTION COEFFICIENT AND NORMALIZED RADAR CROSS SECTION OF TARGET FROM RECORDED DATA

FORTRAN II LANGUAGE

$\mathrm{N}=$ SAMPLED POINTS FROM RECORDED DATA

$\mathrm{R}=$ REFLECTION COEFFICIENT

$C=$ RECORDED SIGNAL FROM WATER-AIR INTERFACE

$D=$ CHANGE OF ATTENUATION IN DECIBLES FOR RECORDED BACKSCATTERED SIGNAL

SIGMA = NORMALIZED RADAR CROSS SECTION

DIMENSION A(500)

DIMENSION $\mathrm{W}(10), \mathrm{X}(10)$

1 READ 2 , N

2 FORMAT (15)

READ 3,FREQ, ANGLE

3 FORMAT (F10,2, F10.2)

READ $4, C, D$

4 FORMAT (F10.2,F10.2)

READ 20, (W (I) , I=1,5), (X (I), I=1,5)

20 FORMAT $(5 \mathrm{Al}, 1 \mathrm{X}, 5 \mathrm{Al})$

READ $21,(A(I), I=1, N)$

21 FORMAT (F 4.0)

PUNCH 100

100 FORMAT $(/ / / / /)$

PUNCH 50, FREQ, ANGLE, (W (I) , $I=1,5),(X(I), I=1,5)$

50 FORMAT $(/ / / / 12$ HFREQUENCY $=, F 7.2,7 \mathrm{X}, 8$ HANGLE $=$,

$1 F 7.2,10 \mathrm{X}, 5 \mathrm{Al}, 1 \mathrm{X}, 5 \mathrm{Al})$

$\mathrm{AN}=\mathrm{N}$

$\mathrm{AVE}=0$.

$S Q A V E=0$.

FOAVE $=0$.

$E=$ LOG $(10) /$.20 .

$\mathrm{Dl}=\mathrm{D} * \mathrm{E}$ 
$\mathrm{Al}=\mathrm{C}{ }^{\star} \operatorname{EXP}(\mathrm{D} 1)$

$\mathrm{A} 2=\mathrm{A} 1$ * $* 2$

$\mathrm{A} 4=\mathrm{A} 2$ * *2

5 DO $6 \quad I=1, N$

$\mathrm{AVE}=\mathrm{AVE}+(\mathrm{A}(\mathrm{I}) / \mathrm{AN})$

SQAVE $=S Q A V E+(A(I) * \star 2 / A N)$

FOAVE=FOAVE+ $(A(I) * * 4 / A N)$

6 CONTINUE

VARCE $=S Q A V E-(A V E *$ * 2$)$

DEVIA $=$ SQRT (ABS (VARCE))

VARSQ $=$ FOAVE - (SQAVE* *2)

DEVSQ $=S Q R T(A B S($ VARSQ) $)$

$\mathrm{AVE}=\mathrm{AVE} / \mathrm{Al}$

SQAVE =SQAVE/A2

VARCE=VARCW/A2

DEVIA $=D E V I A / A I$

VARS $Q=V A R S Q / A 4$

$\mathrm{DEVSQ}=\mathrm{DEVSQ} / \mathrm{A} 2$

PUNCH 16, AVE

16 FORMAT (//12HMEAN OF $R=, F 20.6)$

PUNCH 17 , SQAVE

17 FORMAT $(/ / 16$ HMEAN OF SIGMA $=$, F20.6)

PUNCH 18 , VARCE

18 FORMAT (//16HVARIANCE OF $R=. F 20.6)$

PUNCH 19, DEVIA

19 FORMAT (//26HSTANDARD DEVIATION OF $R=, F 20,6)$

PUNCH 25,VARSQ

25 FORMAT (//20HVARIANCE OF SIGMA $=, F 20.6)$

PUNCH 26, DEVSQ

26 FORMAT (//30HSTANDARD DEVIATION OF SIGMA $=, \quad$ F20.6///)

GO TO 1

END 
APPENDIX II

Comments on Signal Recording; Sample Calculations.

Comments on Recorded Backscattered Signal

Let the voltage recorded on the graphic level recorder be $\mathrm{V}$ volts. Then,

$$
\begin{aligned}
V & =k_{1} E_{r r} \\
& =\frac{k}{r} E_{r}
\end{aligned}
$$

where,

$$
k=a \text { constant. }
$$

$k_{1}=$ the gain of the electronic system between the receiving transducer and the graphic level recorder.

$r=$ the distance of the transducers from the target.

$E_{Y}=$ the voltage proportional to the acoustic pressure scattered by the target (Fig. 14).

$\mathrm{E}_{\mathrm{rr}}=$ the voltage proportional to the backscattered acoustic pressure sensed by the receiving transducer (Fig. 14).

If the reflection coefficient of the target is given by $R$, then

$$
E_{r}=R E_{i}
$$

where, $E_{i}$ is the voltage proportional to the acoustic pressure incident on the target.

Substituting (2) in (1) gives

$$
\mathrm{V}=\frac{\mathrm{k}}{\mathbf{r}} \mathrm{RE}_{\mathbf{i}}
$$




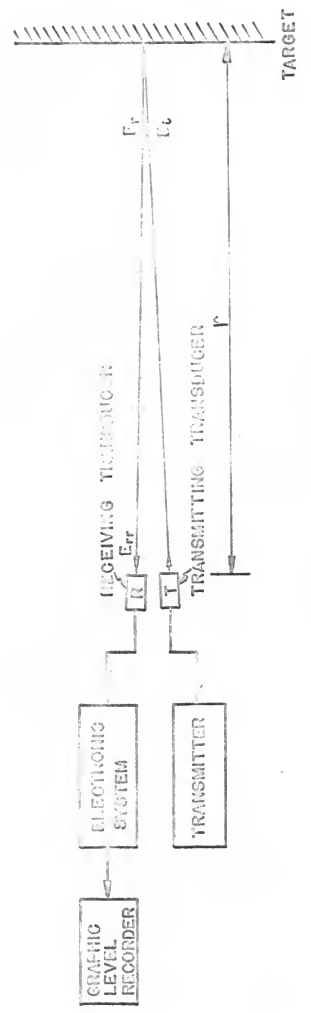

$\frac{1}{2}$
0
$\frac{1}{6}$
$\frac{1}{3}$
$\frac{1}{3}$
0

w
5
5
0
0
$\frac{1}{0}$
0
0

z

$\frac{5}{\frac{5}{4}}$ 
when $R=1$, (3) reduces to

$$
\mathrm{v}_{1}=\frac{\mathrm{k}}{\mathrm{r}} \mathrm{E}_{i}
$$

Dividing (3) by (4) gives

$$
R=\frac{\mathrm{V}}{\mathrm{V}_{1}}
$$

It is well known that

$$
R^{2}=\sigma_{0}
$$

Substituting (6) in (5) gives

$$
\sigma_{0}=\frac{v^{2}}{v_{1}^{2}}
$$

Therefore, if the square of the backscattered signal recorded by the graphic level recorder for any target is divided by the square of the backscattered signal from a water-air interface (for which $R \doteq 1$ ) at the same distance, it will give the normalized radar cross section of the target.

\section{Sample Calculations}

Target: Layer with a rough front.

Backscattered signal: From rough front only.

$f=1.0 \mathrm{Mc}$.

$\theta=0^{\circ}$

$\mathrm{v}_{1}=0.66$ volts. (water-air interface)

Total attenuation present (for $v_{1}=0.6$ volts) in the receiving electronic system $=65 \mathrm{db}$.

Root mean square of backscattered signal from layer $V\left(\left\langle v^{2}\right\rangle\right)=0.286$ volts 
Total attenuation present (for $l\left(\left\langle V^{2}\right\rangle\right)=0.286$ volts) in the receiving electronic system $=50 \mathrm{db}$.

Therefore, the increase in the gain of electronics system to record the backscattered signal from layer $=65-50$ $=15 \mathrm{db}$.

Since the gain of the receiving electronics system was increased while recording the signal backscattered from the layer, the value of $v_{1}$ will have to be modified. Denote this modified value by $\mathrm{v}_{1}^{\prime}$ - Then,

$$
20 \log \frac{v_{1}}{v_{1}}=15 \mathrm{db}
$$

or

$$
\log \frac{v_{1}^{\prime}}{0.66}=\frac{15}{20}=0.75
$$

or

$$
\begin{aligned}
V_{1}^{\prime} & =0.66 \times 5.62 \\
& =3.72 \text { volts } .
\end{aligned}
$$

The value of $\left\langle\sigma_{0}\right\rangle$ is given by

$$
\left\langle\sigma_{0}\right\rangle=\frac{\left\langle V^{2}\right\rangle}{\left(V_{1}{ }^{\top}\right)^{2}}=\frac{(0.286)^{2}}{(3.72)^{2}}=59.2 \times 10^{-4} \text {. }
$$


APPENDIX III

Tables of Measured Data

Table IV. Measured data for rough back configuration of layer.

\begin{tabular}{|c|c|c|c|c|}
\hline $\begin{array}{l}\text { Frequency } \\
\text { in Mc. }\end{array}$ & Ratio $\sigma_{z} / \lambda$ & $\begin{array}{l}\text { Angle of } \\
\text { incidence } \\
\text { in degrees }\end{array}$ & $\left\langle\sigma_{0}\right\rangle$ & $\begin{array}{l}\text { Standard } \\
\text { deviation of } \\
\left\langle\sigma_{0}\right\rangle\end{array}$ \\
\hline 0.72 & 0.306 & $\begin{array}{r}0 \\
5 \\
10 \\
\end{array}$ & $\begin{array}{l}0.008632 \\
0.008300 \\
0.008893 \\
\end{array}$ & $\begin{array}{l}0.006411 \\
0.005937 \\
0.005540\end{array}$ \\
\hline 1.00 & 0.425 & $\begin{array}{r}0 \\
5 \\
10 \\
20 \\
\end{array}$ & $\begin{array}{l}0.002217 \\
0.001208 \\
0.001666 \\
0.000340 \\
\end{array}$ & $\begin{array}{l}0.002229 \\
0.001078 \\
0.001995 \\
0.000288 \\
\end{array}$ \\
\hline 1.28 & 0.544 & $\begin{array}{r}0 \\
5 \\
10 \\
\end{array}$ & $\begin{array}{l}0.001203 \\
0.001116 \\
0.001249 \\
\end{array}$ & $\begin{array}{l}0.000660 \\
0.000867 \\
0.001040 \\
\end{array}$ \\
\hline 1.60 & 0.679 & $\begin{array}{r}0 \\
5 \\
10 \\
20 \\
\end{array}$ & $\begin{array}{l}0.000480 \\
0.000638 \\
0.001018 \\
0.000221 \\
\end{array}$ & $\begin{array}{l}0.000427 \\
0.000674 \\
0.000957 \\
0.000190 \\
\end{array}$ \\
\hline 1.90 & 0.806 & $\begin{array}{r}0 \\
5 \\
10 \\
\end{array}$ & $\begin{array}{l}0.000166 \\
0.000168 \\
0.000269 \\
\end{array}$ & $\begin{array}{l}0.000114 \\
0.000131 \\
0.000241 \\
\end{array}$ \\
\hline 2.25 & 0.955 & $\begin{array}{r}0 \\
5 \\
10 \\
20 \\
\end{array}$ & $\begin{array}{l}0.000111 \\
0.000158 \\
0.000273 \\
0.000046 \\
\end{array}$ & $\begin{array}{l}0.000116 \\
0.000132 \\
0.000210 \\
0.000046\end{array}$ \\
\hline 3.00 & 1.270 & $\begin{array}{r}0 \\
5 \\
10 \\
\end{array}$ & $\begin{array}{l}0.000093 \\
0.000014 \\
0.000019 \\
\end{array}$ & $\begin{array}{l}0.000044 \\
0.000008 \\
0.000010\end{array}$ \\
\hline 3.50 & 1.490 & $\begin{array}{r}0 \\
5 \\
10 \\
20\end{array}$ & $\begin{array}{l}0.000054 \\
0.000009 \\
0.000004 \\
0.000000\end{array}$ & $\begin{array}{l}0.000079 \\
0.000007 \\
0.000004 \\
0.000000\end{array}$ \\
\hline
\end{tabular}


Table V. Measured data for rough front configuration of layer

\begin{tabular}{|c|c|c|c|c|}
\hline $\begin{array}{l}\text { Frequency } \\
\text { in Mc, }\end{array}$ & Ratio $\sigma_{z} / \lambda$ & $\begin{array}{l}\text { Angle of } \\
\text { incidence } \\
\text { in degrees }\end{array}$ & $\left\langle\sigma_{0}\right\rangle$ & $\begin{array}{l}\text { Standard } \\
\text { deviation } \\
\text { of }\left\langle\sigma_{0}\right\rangle\end{array}$ \\
\hline 1.00 & 0.787 & $\begin{array}{r}0 \\
5 \\
10 \\
20 \\
\end{array}$ & $\begin{array}{l}0.006088 \\
0.003782 \\
0.003544 \\
0.001468 \\
\end{array}$ & $\begin{array}{l}0.006694 \\
0.006298 \\
0.006640 \\
0.002024 \\
\end{array}$ \\
\hline 1.60 & 1.250 & $\begin{array}{r}0 \\
5 \\
10 \\
20\end{array}$ & $\begin{array}{l}0.001422 \\
0.000597 \\
0.001910 \\
0.000423\end{array}$ & $\begin{array}{l}0.001497 \\
0.000454 \\
0.002149 \\
0.000590 \\
\end{array}$ \\
\hline 2.25 & 1.770 & $\begin{array}{r}0 \\
5 \\
10 \\
20 \\
\end{array}$ & $\begin{array}{l}0.001353 \\
0.002490 \\
0.002564 \\
0.001067 \\
\end{array}$ & $\begin{array}{l}0.000852 \\
0.001563 \\
0.002345 \\
0.001899 \\
\end{array}$ \\
\hline 3.50 & 2.760 & $\begin{array}{r}0 \\
5 \\
10 \\
20 \\
\end{array}$ & $\begin{array}{l}0.000651 \\
0.000680 \\
0.000715 \\
0.000388 \\
\end{array}$ & $\begin{array}{l}0.000424 \\
0.000417 \\
0.000487 \\
0.000589\end{array}$ \\
\hline
\end{tabular}




\section{APPENDIX IV}

Scattering from Periodically Rough Surfaces.

The equation from grating theory, given below, indicates that a periodic surface scatters the incident wave into a number of different modes (or lobes) as compared to a rough surface which scatters diffusely.

$$
\sin \theta_{2 m}=\sin \theta_{1}+m \frac{\lambda}{\Lambda} \quad(m=0, \pm 1,+2, \ldots)
$$

where

$$
\begin{aligned}
& { }_{1}^{\theta_{1}}=\text { angle of incidence } \\
& \lambda=\text { wavelength of incident wave- } \\
& \Lambda=\text { wavelength of periodic surface } \\
& m=\text { any integer } \\
& { }^{\theta_{2}}=\text { scattering angle corresponding to each mode. }
\end{aligned}
$$

The scattering angles $\theta_{2 \mathrm{~m}}$ are determined by the grating equation ( 1 ). To each integer $\mathrm{m}$ there corresponds a scattered mode propagated in the direction $\theta_{2 \mathrm{~m}}$. The total number of possible modes is limited by the condition.

$$
\left|\sin \theta_{2 m}\right| \leq 1
$$

The mode $m=0$ is seen from ( 1 ) to be the specular mode. The modes $m= \pm 1$ lie to either side of the specular direction. The modes continue thus to either side of the specular mode until the last modes that will satisfy (2) are reached.

If $\lambda / \Lambda$ is small, it follows from (1) that $m$ will run through a large number of integral values before (2) is violated, so that 
if the wavelength of the incident radiation is small compared to the period or wavelength of the surface, the incident wave will be broken up into many scattered waves. 


\section{BIBLIOGRAPHY}

1. Ament, W. S. Toward a Theory of Reflection By a Rough Surface. Proc. I.R.E. $41,142-146 ; 1953$.

2. Ament, W. S. Reciprocity and Scattering By Certain Rough Surfaces. Trans. I.R.E. AP-8, 167-174; 1960 .

3. Albers, V. M. Underwater Acoustics Handbook. The Pennsylvania State University , Press, University Park, Pennsylvania, 1965.

4. Beckmann, P.

The Scattering of Waves From a Periodic Surface. Czechosl. J. Phys., 196ia.

5. Beckmann, P. and Spizzichino, A. The Scattering of Electromagnetic Waves from Rough Surfaces. Pergamon Press, Macmillan Company, New York, 1963.

6. Beckmann, P.

Scattering by Composite Rough Surfaces. Proc. I.E.E.E., Vol. 53, pp. 1012-1015; August, 1965.

7. Blitz, J. Fundamentals of Ultrasonics. Butterworth and Company, London, 1963.

8. Born, M. and Wolf, E. Principle of Optics, Pergamon Press, New York, pp. 383$384 ; 1959$.

9. Brekhovskikh, L. M.

Waves in Layered Media. Academic Press, New York and London, 1960.

10. Carlin, B.

Ultrasonics. McGraw-Hill Book Company, Inc., 1960.

11. Clarke, R. H.

Theoretical Characteristics of Radiation Reflected Obliquely from a Rough Conducting Surface. Proc. I.E.E., Vol. 110, pp. 91-100; January, 1963.

12. Clarke, R. H.

Some Measurements on Radiation Reflected Obliquely From a Rough Surface. Proc. I.E.E., Vol. 110, pp. 1921-1927; November, 1963.

13. Clarke, R. H. and Hendry, G. O.

Prediction and Measurement of the Coherent and Incoherent 
Power Reflected from a Rough Surface. I.E.E.E. Transactions on Antennas and Propagation, Vol. AP-12, pp. 353-363; May, 1964 .

14. Davies, H.

The Reflection of Electromagnetic Waves From a Rough Surface. Proc. I.E.E. Pt. III 101, 209-214; 1954.

15. Deryugin, L. N.

The Reflection of a Longitudinally Polarized Plane Wave From a Surface of Rectangular Corrugations (in Russian), Radiotekhn. 15, No. 5, 9-16; $1960 \mathrm{~b}$.

16. Eckart, C.

The Scattering of Sound From Sea surface. J. Acoustic Society of America, Vol. 25; pp. 566-570, 1953.

17. Evans, J. V. and Pettengill, G. H. The Scattering Behaviour of the Moon at Wavelengths of $3.6,68$ and 784 centimeters. Journ. Geo. Res., Vol. 68 , No. 2, Jan. 15,1963 .

18. Evans, J. V. and Pettengill, G. H. The Scattering Properties of the Lunar surface of Radio Wavelengths. The Solar System, Vol. IV. Univ. of Chicago Press, pp. 129-161, 1963.

19. Fung, A. K. Scattering Theories and Radar Return. CRES Report No. 48-3, The University of Kansas, Center for Research Inc., Engineering Science Division, Lawrence, Kansas.

20. Gjessing, D. T. and Irgens, F. Scattering of Radio Waves by a Moving Atmospheric Rippled Layer: A Simple Model-Experiment. I.E.E.E. Transactions on Antennas and Propagation, Vol. AP-12, pp. 703-709; November 1964 .

21. Hagfors, T. (et.al.)

Evidence of a Tenuous Surface Layer on the Moon as Derived From Radar observations, Presented to Science:, M.I.T., July, 1965.

22. Hueter, T. F. and Bolt, R. H. Sonics, John Wiley and Sons, Inc., New York, 1955.

23. Isakovich, M. A. The Scattering of Waves From a Statistically Rough Surface (in Russian). Zhwn. Eksp. Teor. Fiz. 23, pp. 305-314, 1952.

24. Kaufman, D. E. and Hayre, H. S.

Electromagnetic Wave Scattering From Very Rough surfaces Composed of Correlated Large and Small scale Roughness. Proc. I.E.E.E. pp. 1157-58; August, 1965. 
25. Kerr, D. E. (Editor)

Propagation of Short Waves, McGraw-Hill, 1964.

26. Krasil'nikov, V. A.

Sound and Ultrasound Waves in Air, Water and Solid Bodies (Translated from Russian), Israel Program for scientific Translations, Jerusalem, 1963.

27. Middleton, D.

An Introduction to Statistical Communication Theory. McGraw-Hill Book Company, Inc., 1960.

28. Muhleman, D. O., Goldstein, R., and Carpenter, R. A review of Radar Astronomy-Part I. I.E.E.E. Spectrum pp. 44-55; October 1965 .

29. Muhleman, D. O., Goldstein, R. and Carpenter, R. A review of Radar Astronomy-Part II. I.E.E.E. Spectrum pp. 78-89; November, 1965 .

30. Parks, J. K. Toward a Simple Mathematical Model for Microwave Backscatter from the Sea Surface at Near Vertical Incidence. I.E.E.E. Transactions on Antennas and Propagation, Vol. AP-12, pp. 590-605; September, 1964.

31. Parkins, B. E. The Omnidirectional scattering of Acoustic Waves from Rough Surfaces with Application to Electromagnetic Scatteringi, CRES Report No. 48-4. The University of Kansas, Center for Research Inc., Engineering Science Division, Lawrence, Kansas.

32. Richardson, E. G.

Analogies Between Acoustic and Electromagnetic Phenomenon. Brit. I.R.E., Vol. 12, pp. 577-84; November 1952.

33. Ridenour, L. S.

Radar System Engineering. McGraw-Hill Book Company, Inc., 1947.

34. Senior, T.B.A.

Surface Roughness and Tolerances in Model Scattering Experiments. I.E.E.E. Trans. on Antennas and Propagation, Vol. AP-13, pp. 629-636; July, 1965.

35. Silver, $\mathrm{S}$.

Microwave Antenna Theory and Design. McGraw-Hill Book

Company, Inc., 1949.

36. Skolnik, M. I.

Introduction to Radar Systems. McGraw-Hill Book Co., Inc. 1962 . 
37. Stratton, J.A.

Electromagnetic Theory. McGraw-Hill Book Co., Inc., 1941.

38. Taylor, R. C.

Terrain Return Measurements at $\mathrm{x}, \mathrm{k}_{\mathrm{u}}$, and $\mathrm{k}_{2}$ band. presented at Nat. I.R.E. Meeting in New York, 1959.

Antenna Lab. The Ohio State University, Columbus, Ohio.

39. Toliver, J. W., Kaufman, D. E. and Durrani, S. H.

The K. S. U. Acoustic Simulator for Radar Studies.

Technical Report EE-TR-1, Dept. of Elec. Engg., Kansas

State University, Manhattan, Kansas.

40. Twersky, V.

On the Scattering and Reflection of Electromagnetic

Waves by Rough Surfaces. Trans. I.R.E., Vol. AP-5, pp. $81-90 ; 1957 a$.

41. Twersky, V.

On Scattering and Reflection of Sound By Rough Surfaces. Journ. Ac. Soc. Am., Vol. 29, pp. 209-225; $1957 \mathrm{~b}$. 
BACKSCATTER OF ELECTROMAGNETIC WAVES

FROM A ROUGH LAYYR

by

VIJAY RAJ KUMAR

B. S., Banaras Hindu University, 1961

AN ABSTRACT OF A MASTER'S THESIS

submitted in partial fulfillment of the

requirements for the degree

MASTER OF SCIENCE

Department of Electrical Engineering

KANSAS STATE UNIVERSITY

Manhattan, Kansas

1966 
The radar cross section of a rough surface is often used to predict the dielectric and statistical properties of the scatterer. In an attempt to extend this approach, a target consisting of a rough layer with the front surface smooth and the back surface statistically rough was chosen for this study. Theoretical expressions were derived for the mean value of the radar cross section $\left\langle\sigma_{0}\right\rangle$ of the rough layer. These expressions for $\left\langle\sigma_{0}\right\rangle$ turn out to be complicated functions of the angles of incidence and scatter, frequency, mean depth of layer, dielectric and statistical properties of the rough layer, etc.

To better understand the behaviour of a rough layer, experimental work was conducted using the acoustic simulation technique. A plexiglas layer 48 inches long, 6 inches wide and 2 inches thick, was carefully machined on one surface to obtain a statistical variation in the thickness with nearly Gaussian distribution and nearly Gaussian correlation function. The layer was placed in a water tank with water on one side and air on the other, and was struck by pressure waves from electro-acoustic transducers of different frequencies, in the usual manner.

The backscattered signal was recorded for eight frequencies, four angles of incidence and two different configurations of the layer:

(a) The front surface smooth and the back surface rough.

(b) The front surface rough and the back surface smooth. From the measured data, the following curves were drawn: 
(1) $\left\langle\sigma_{0}\right\rangle$ versus $\sigma_{z} / \lambda$, the back interface being rough.

(2) $\left\langle\sigma_{0}\right\rangle$ versus angle of incidence, the back interface being rough.

(3) Same as (1), but with the front interface rough.

(4) Same as (2), but with the front interface rough. Here $\sigma_{z}$ and $\lambda$ are the standard deviation of the heights of the rough surface of layer, and the wavelength of the incident wave, respectively.

Only a partial attempt was made to check the experimental results with those obtained from theory, due to certain limitations of the experimental data. The theoretical as well as experimental results indicate that the average radar cross section $\left\langle\sigma_{0}\right\rangle$ of a layered target decreases as the rough surface of layer becomes more rough (i.e. $\sigma_{z} / \lambda$ increases), and as the angle of incidence becomes more oblique. Other implications of the theoretical expressions and the measured curves are also discussed. 